
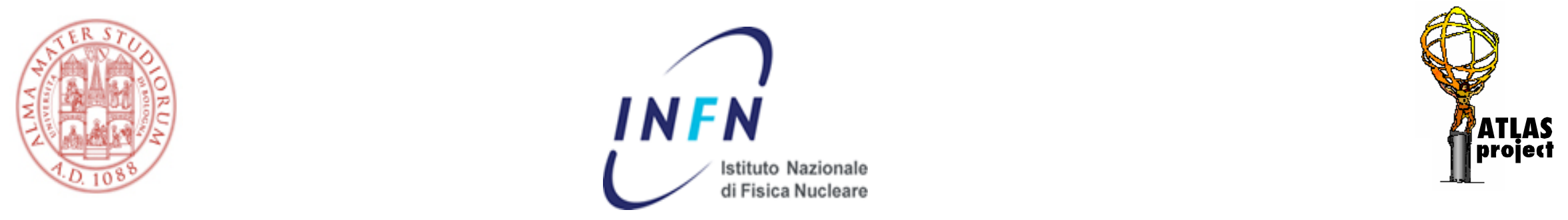

\title{
The luminosity monitor of the ATLAS experiment
}

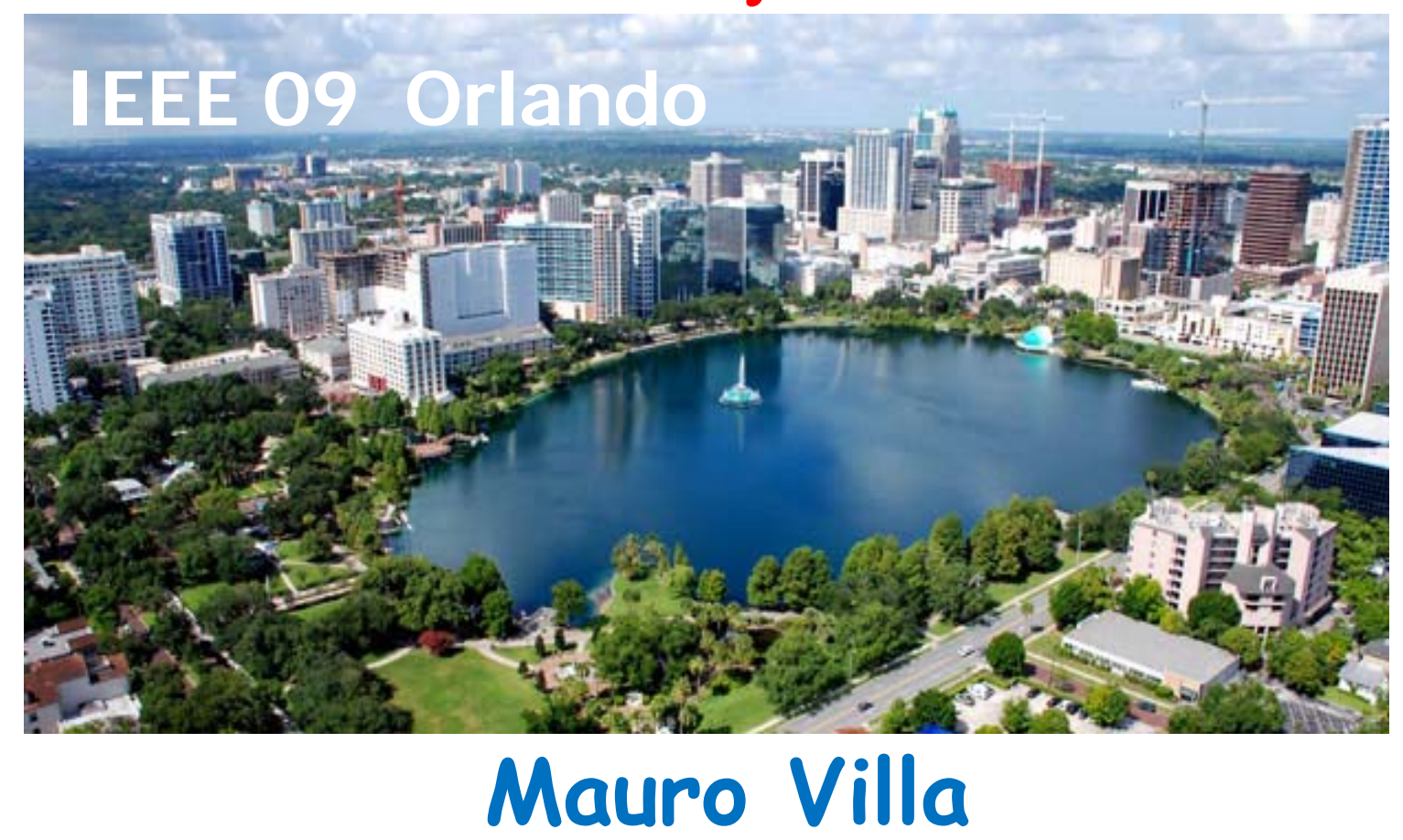

Bologna University and INFN (IT)

On behalf of the LUCID group in ATLAS

[Alberta (CD), CERN (CH), Lund (SW), Bologna (IT)] 


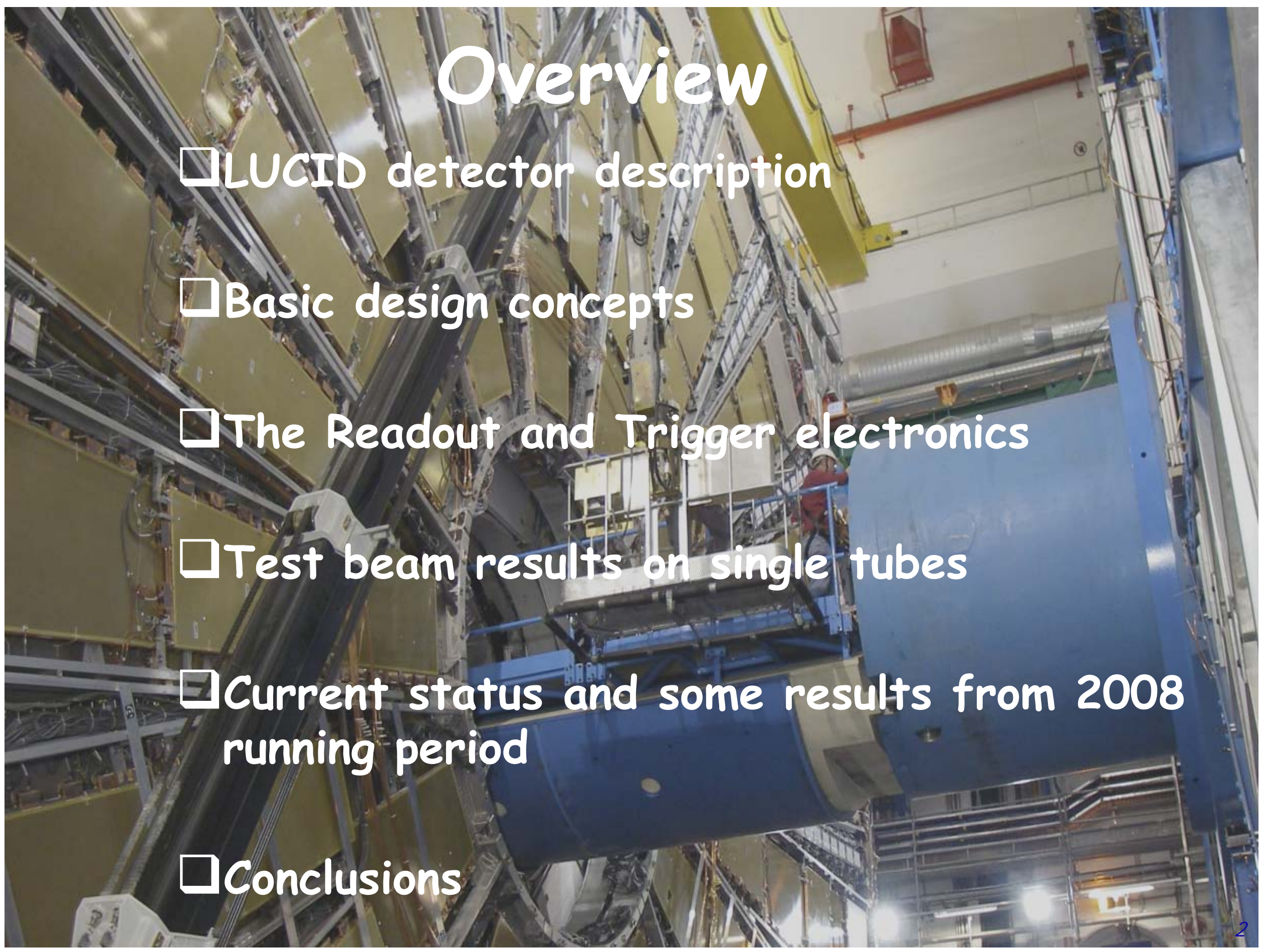




\section{LUCID location and purposes}

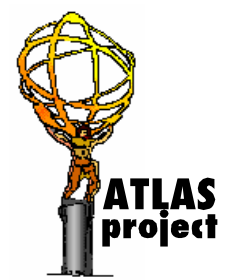

LUCID: LUminosity Cherenkov Integrating Detector

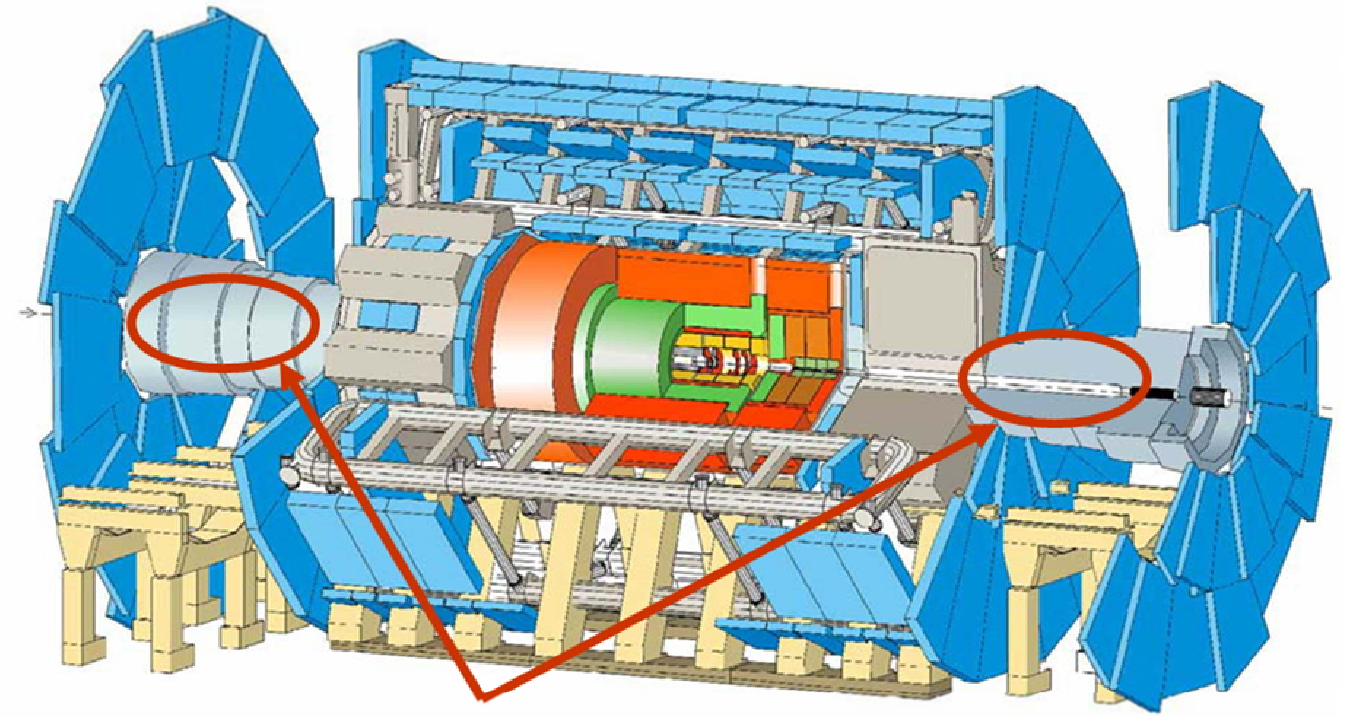

Location of LUCID modules inside ATLAS
- LUCID is made of two modules located at $17 \mathrm{~m}$ from the interaction point

- LUCID is designed to measure the luminosity up to $L=4 \times 10^{33} \mathrm{~cm}^{-2} \mathrm{~s}^{-1}$

- Sensitive to charged particles pointing to the primary $p p$ collisions

$\square$ Array of gaseous Cherenkov detectors having the following purposes:

- Measure and monitor the ATLAS RELATIVE LUMINOSITY integrated over the time and for each Bunch Crossing

- Provide an INTERACTION TRIGGER

$\square$ When calibrated, LUCID will provide the ATLAS ABSOLUTE LUMINOSITY measurement 


\section{LUCID detector principle}

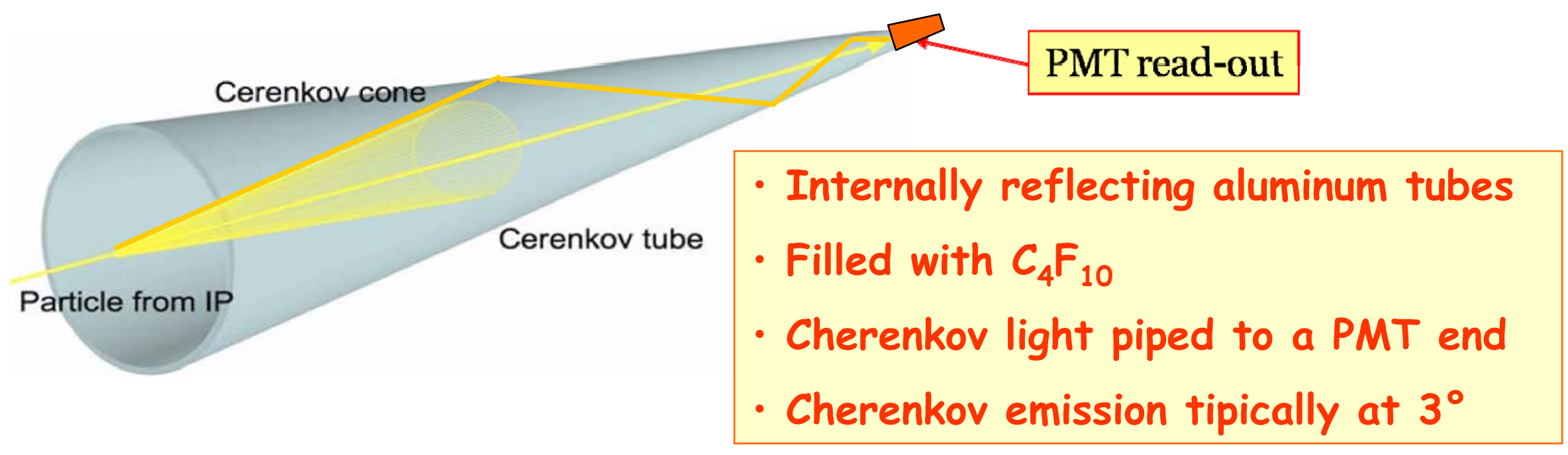

$\checkmark$ Fast detector responce (few ns)

$\checkmark$ allows for single bunch crossing detection (25 ns spacing)

$\checkmark$ Light design: intrinsically radiation tolerant

$\checkmark$ Background suppression

$\checkmark$ Cherenkov threshold: $2.8 \mathrm{GeV} / \mathrm{c}$ for $\pi$ in gas

$\checkmark$ Geometry: tubes are pointing to the $p p$ interaction region 


\section{Detector Description}
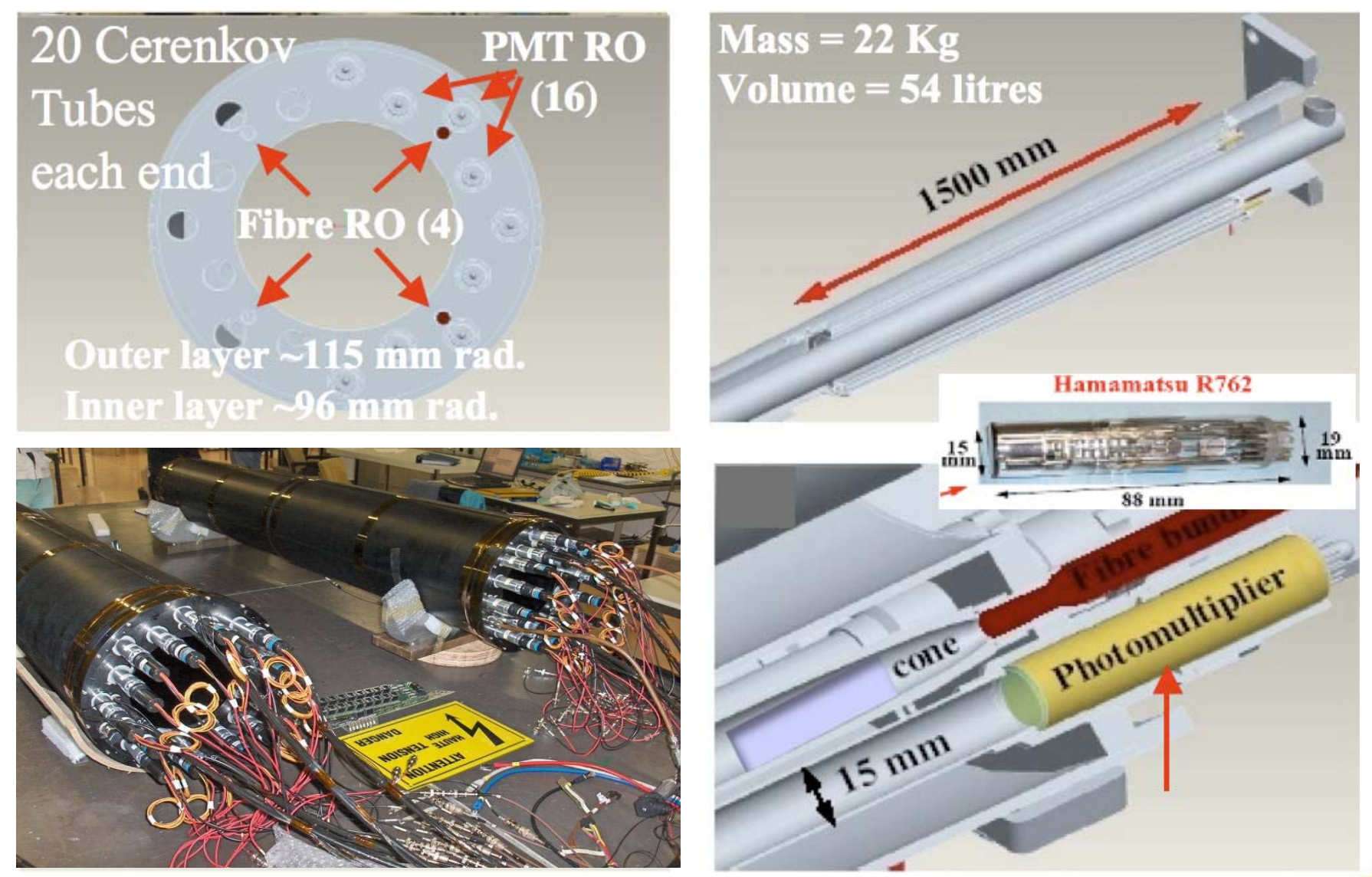

$\square 20$ Pointing Cherenkov tubes on each side of ATLAS IP, filled with $C_{4} F_{10}$ radiator

$\square$ LUCID eta coverage $5.61<\eta<5.93$ Mauro Villa - IEEE 09 - Orlando

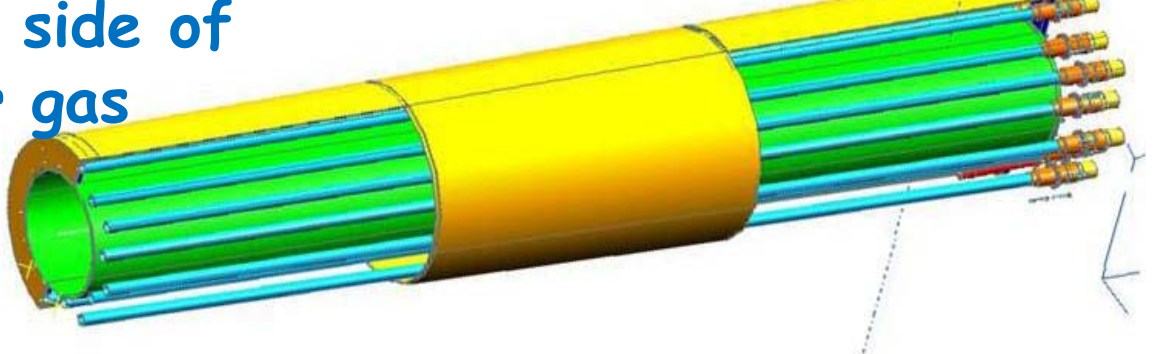




\section{LUCID Readout Scheme}

$2 \times 16$ tubes are directly coupled to photomultipliers (PMT)

PMT must stand the high radiation environment (0.7 MRad/y)
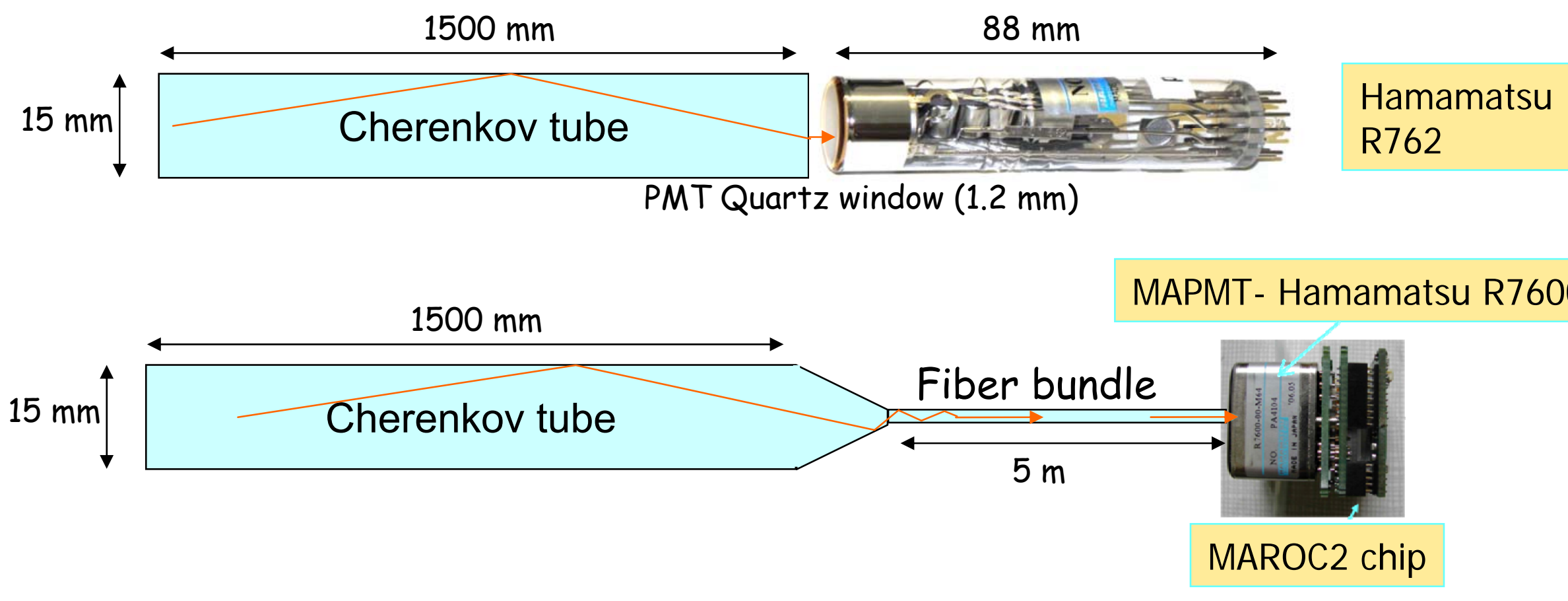

$2 \times 4$ tubes are coupled to a multi-anode PMT via Aluminium cones and optical fibers Better for high luminosity runs (MAPMT not exposed to high radiation doses) 


\section{Gamma and Neutron irradiation tests}

$y:{ }^{60} \mathrm{Co}, E=1.22 \mathrm{MeV}$

Total dose: $20 \pm 1 \mathrm{MRad}$ in $22 \mathrm{~h}$ 30 years of $L H C$ in phase $I$

$\checkmark$ No effects on gain and on spectral responce:

$\checkmark$ Increase of dark current

$\checkmark$ Darkening of the glass, no change on the quartz window

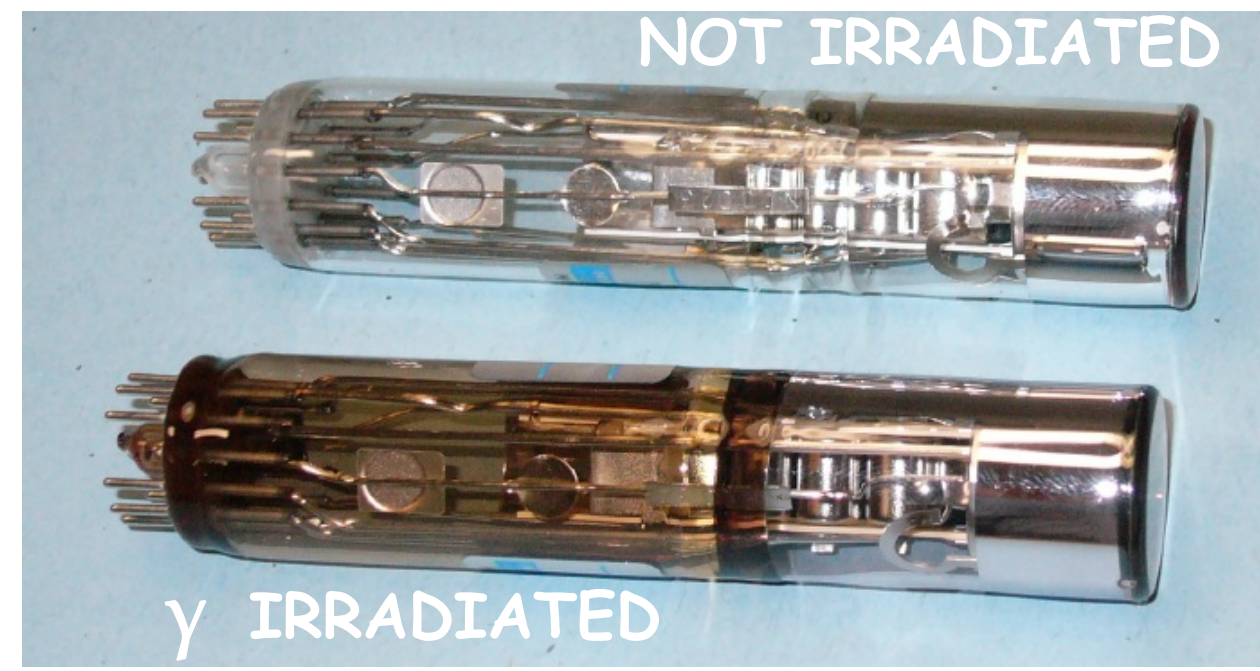

Mauro Villa - IEEE 09 - Orlando n: ENEA Casaccia Reactor

$E=100 \mathrm{keV}$ (average)

$1.5 \mathrm{~h}$ at $5 \times 10^{14} \mathrm{n} / \mathrm{s}$

total dose equivalent of 20 years of LHC in phase I

$\checkmark$ No effects on gain and on spectral responce;

$\checkmark$ Increase of dark current

$\checkmark$ Activation of short lived nuclei (Al, 12min )

No relevant radiation effects foreseen during the first years of LHC running (low lumi) 


\section{The LUCID readout DATA FLOW}

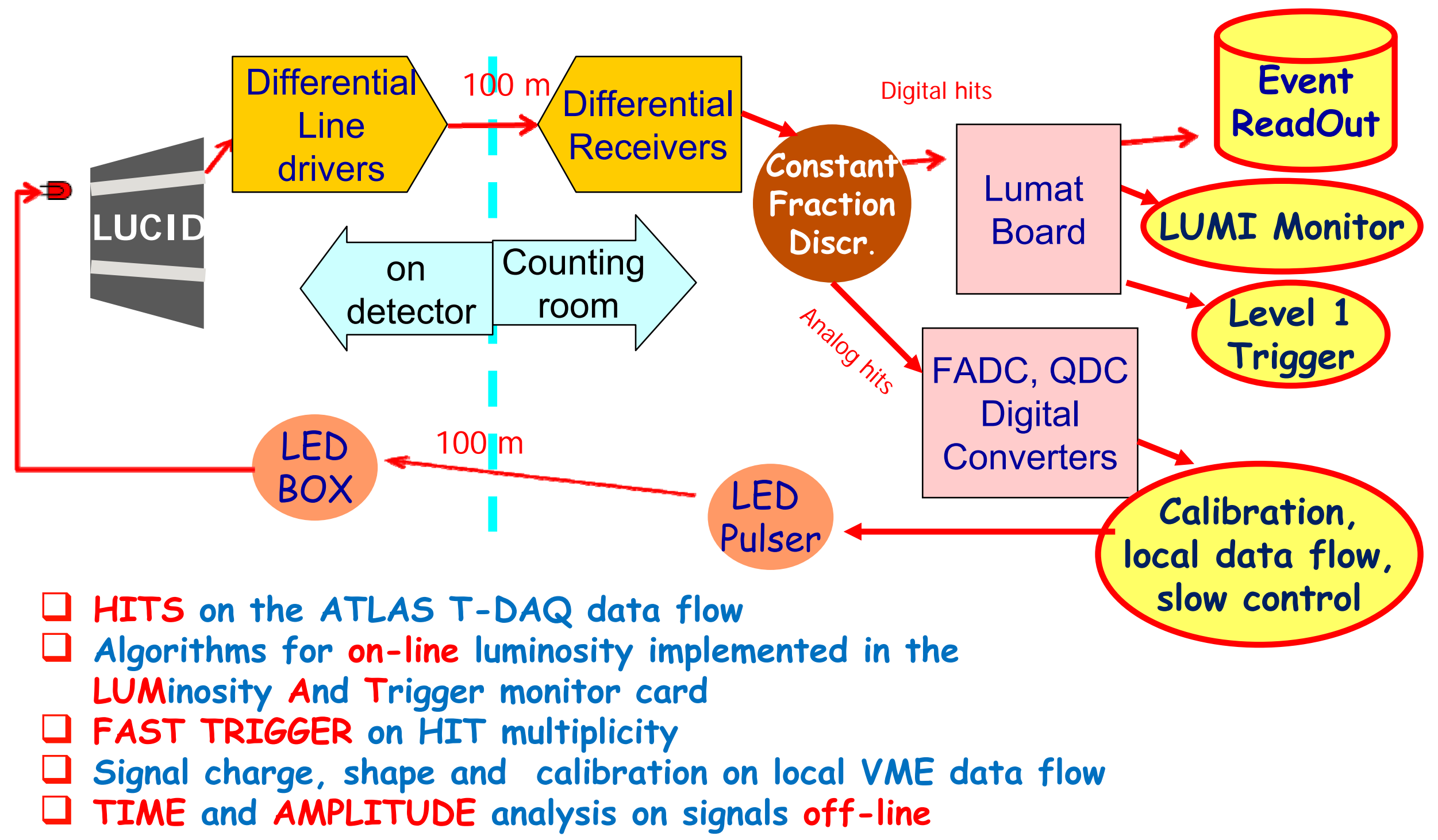




\section{LED CALIBRATION DATA}

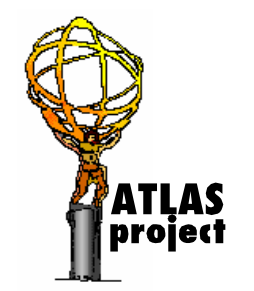

Signal $\mathrm{Q}_{1}$ : $17-45 \mathrm{ch} / \mathrm{pe}$

1 photo electron (pe) peak

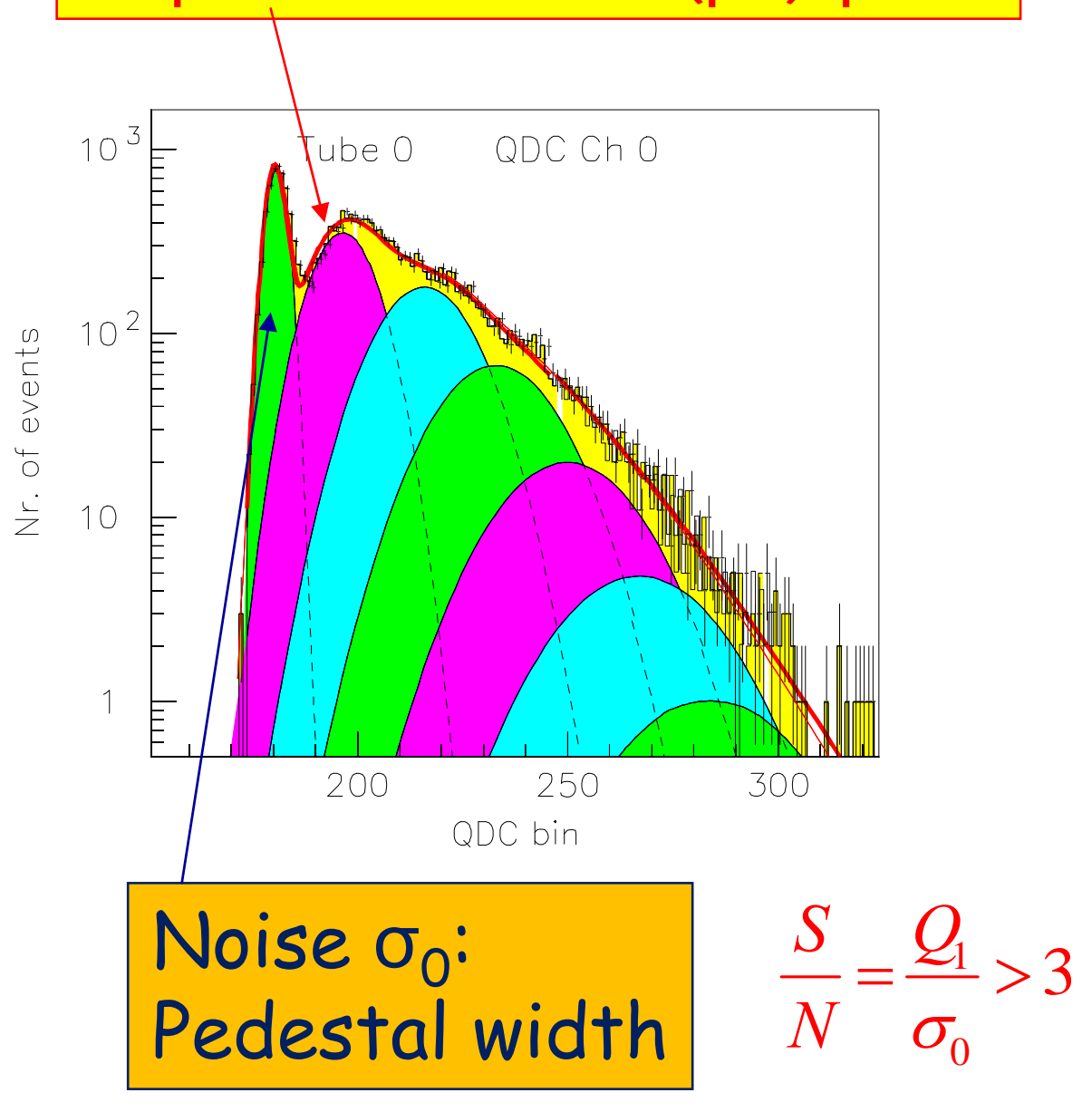

Single photoelectron signal after $\sim 100 \mathrm{~m}$ cable LUCID READS SINGLE BUNCHES!

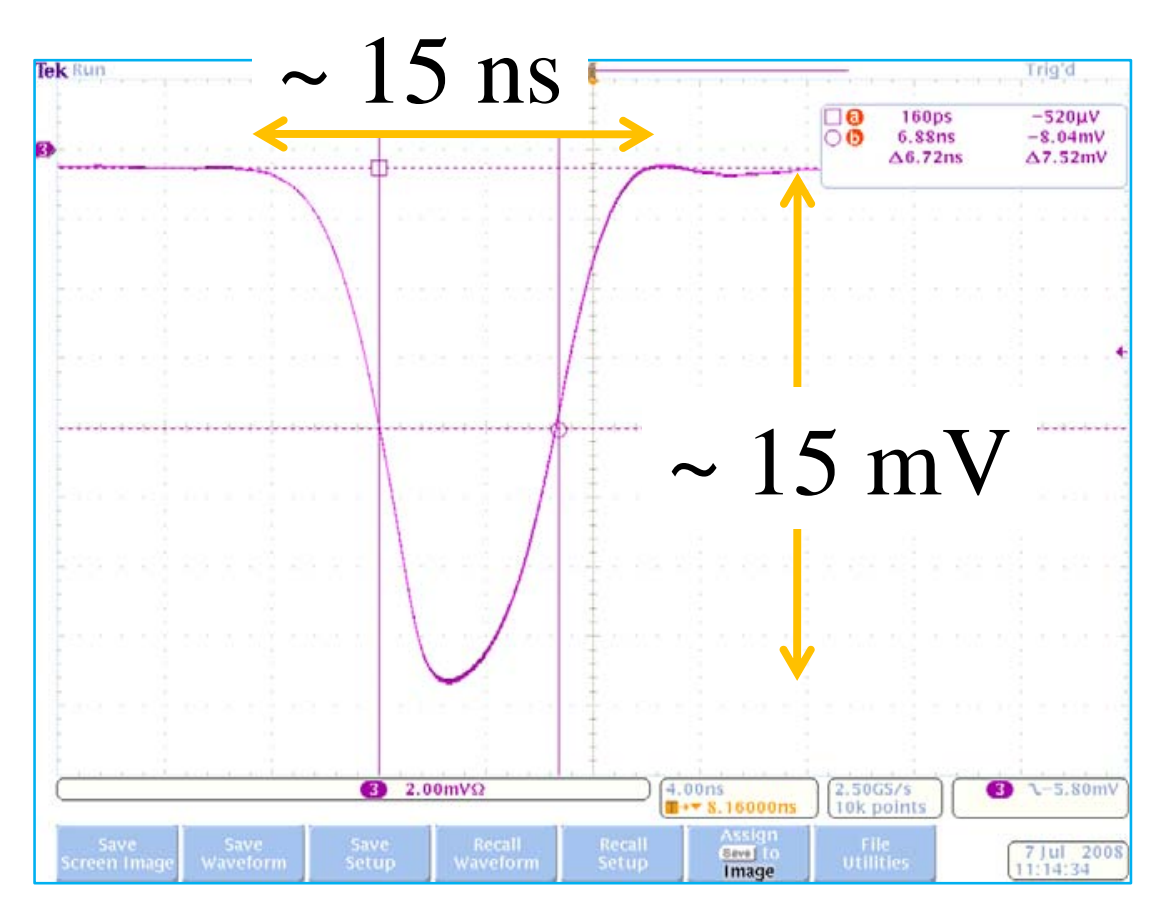

Typical 1 track signal 70 pe 


\section{LUminosity Monitor And Trigger card}

$\checkmark 9 U$ VME Board

$\checkmark 5$ piggy back boards

$\checkmark 4$ Fast FPGAs

$\checkmark$ Programmability

$\checkmark$ Flexibility

Performances:

$40 \mathrm{Mhz}$ bus clocks $8 \mathrm{Gbit} / \mathrm{s}$ input rate $1 \mathrm{Gbit} / \mathrm{s}$ output rate

Goals

$\checkmark$ Luminosity evaluation * Several algorithms

$\checkmark$ LUCID event DAQ

$\checkmark$ LUCID Monitoring

$\checkmark$ LUCID Triggers

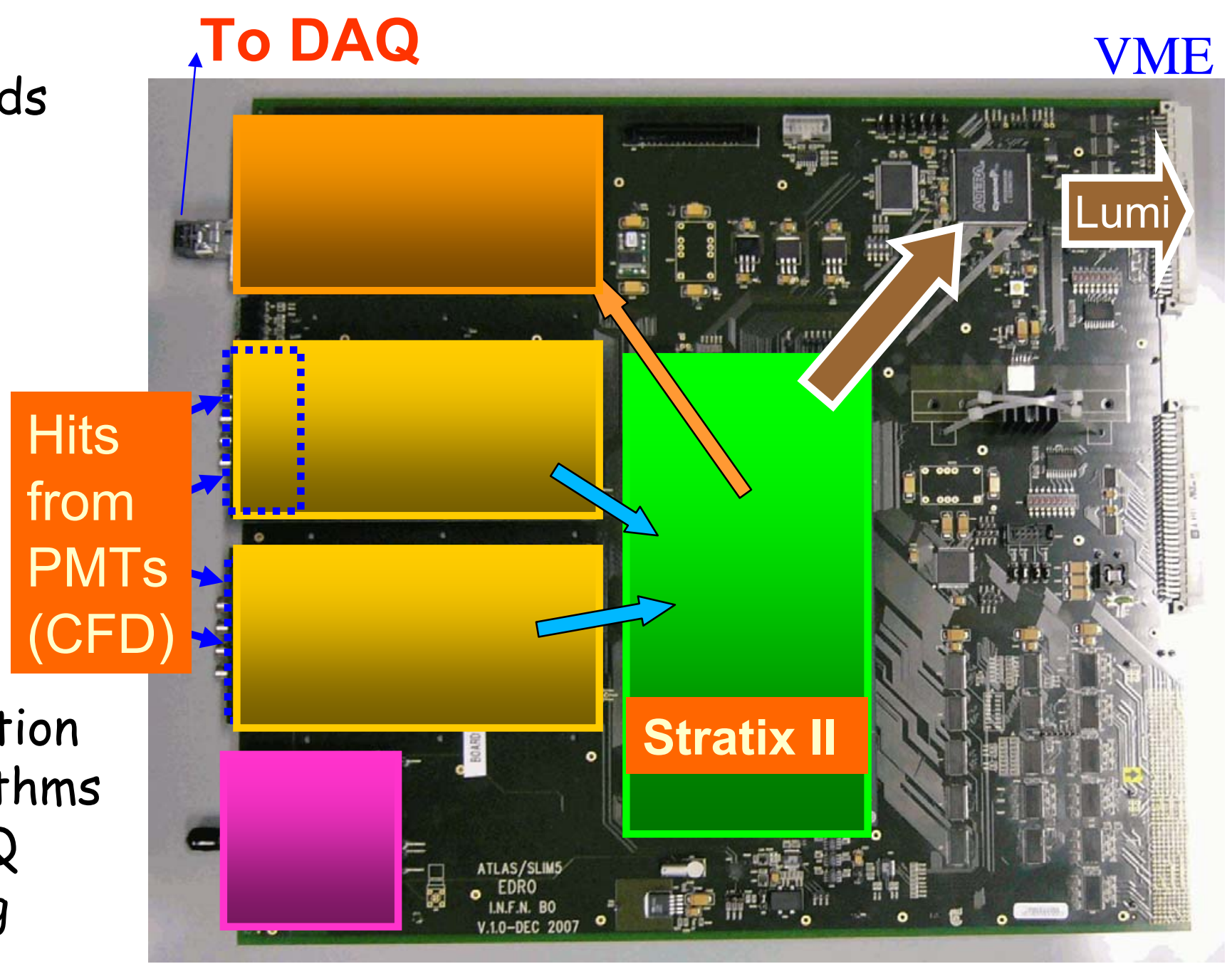




\section{Methods to measure luminosity}

The rate of the pp interactions $\left(R_{p p}\right)$ seen by LUCID is proportional to the luminosity $(L)$ :

filled $\mathrm{BX}$

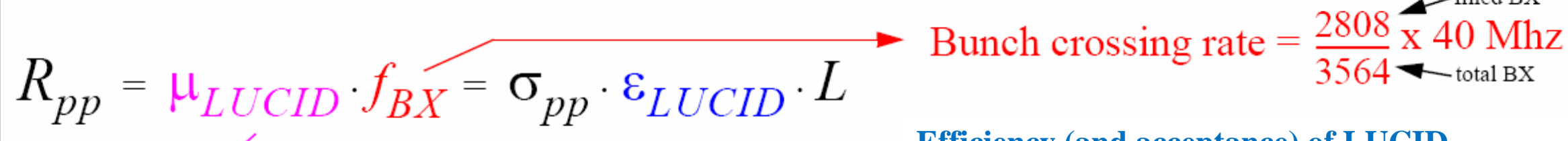
Efficiency (and acceptance) of LUCID $\longrightarrow$ To detect a pp interaction

Number of pp interactions per bunch-

crossing (BX) as measured by LUCID.

\section{Zero Counting \\ On and Off-line}

Count bunch crossings with no interactions:

$$
\begin{aligned}
\mu_{\mathrm{LUCID}} & =-\ln \left(\frac{N_{\text {zero } B X}}{N_{\text {totalBX }}}\right) \\
\mu_{\mathrm{LUCID}}= & \frac{<\mathrm{N}_{\text {hits } / \mathrm{BX}}>}{<\mathrm{N}_{\text {hits } / \mathrm{pp}}>} \\
\mu_{\mathrm{LUCID}}= & \frac{<\mathrm{N}_{\text {particles } / \mathrm{BX}}>}{<\mathrm{N}_{\text {particles } / \mathrm{pp}}>}
\end{aligned}
$$

Hit Counting

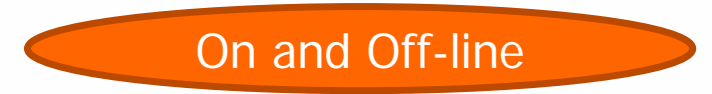

Particle Counting

Off-line

Count the number of particles in LUCID by doing several cuts on the pulseheight distributions: 


\section{Hardware simulation of LHC beams}

$\checkmark$ Waiting for the data, we have an hardware beam simulator:

Luminosity on

A bunch-by-bunch

Basis (25 ns)

A grand total of 6 luminosity algorithms available

Luminosity measured every 0.1-10 minutes (lumi block) for all the time

Algorithms and infrastructures ready for real data!
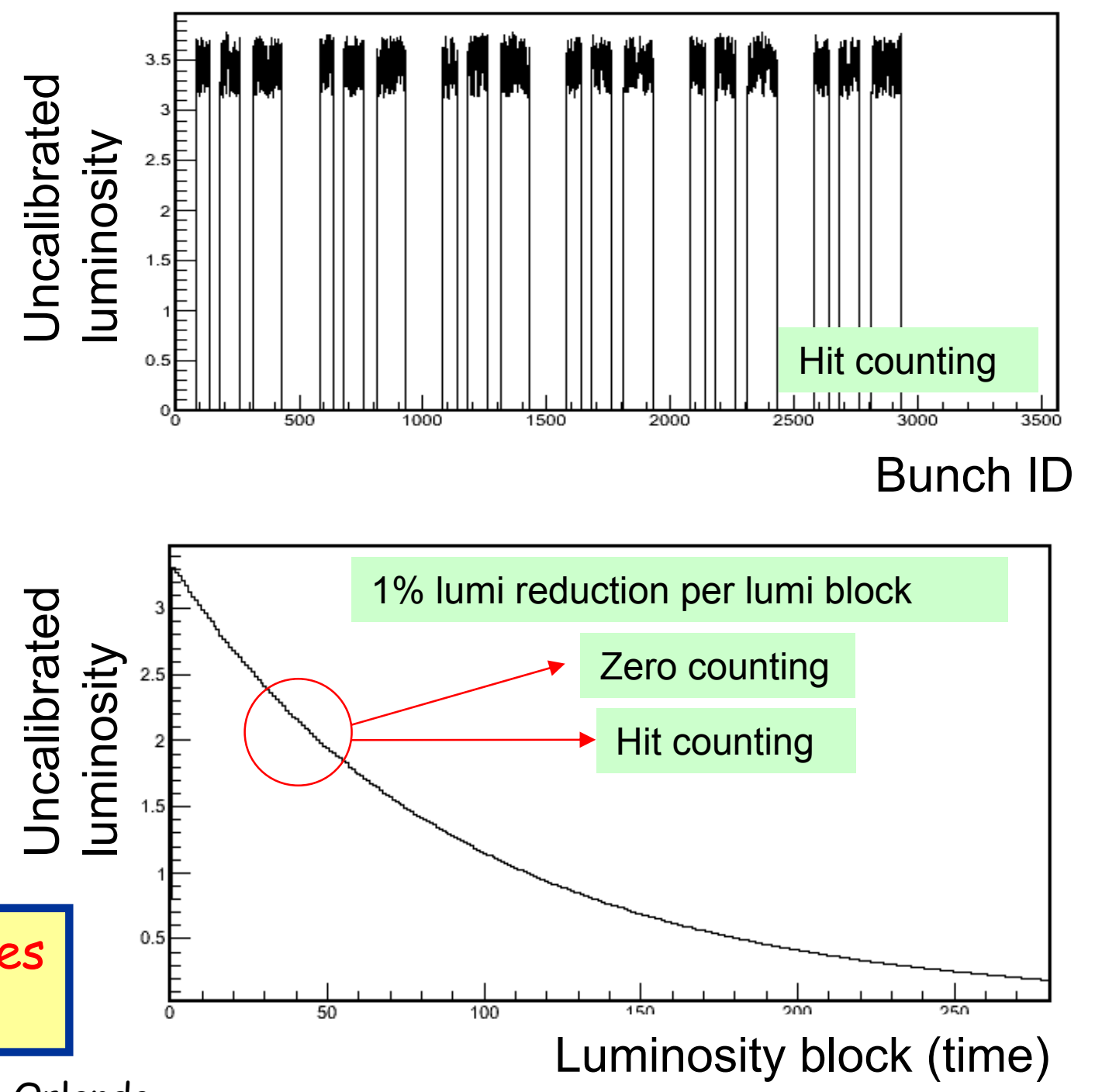

Mauro Villa - IEEE 09 - Orlando 


\section{LUCID beam tests}

$\square$ A systematic test of tubes and gases has been performed at the H6 SPS beam line: pions, $120 \mathrm{GeV}, 2-3 \times 10^{5} \mathrm{~m}^{+} /$spill

$\square$ Gas studied: $C_{4} F_{10}$. Isobutane, Nitrogen. Pressure 150 to 1800 mbar

$\square$ External trigger on scintillators

$\square$ Tracks measured with a microstrip system (SLIM5 exp.)

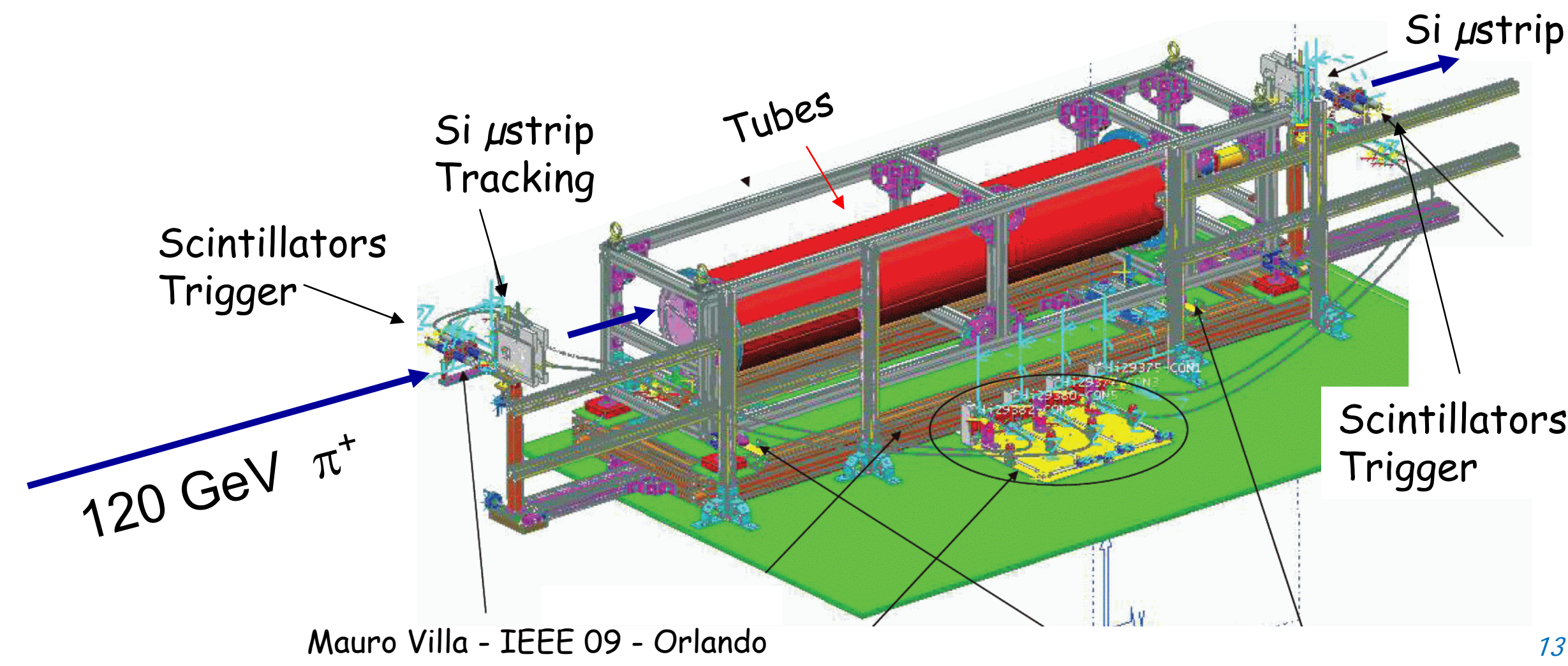




\section{Signals types}

$\square$ Two cherenkov contributions: in the PMT quartz window and in gas

$\square$ PMT contribution constant over pressure and angles $\left(|\theta|<10^{\circ}\right)$
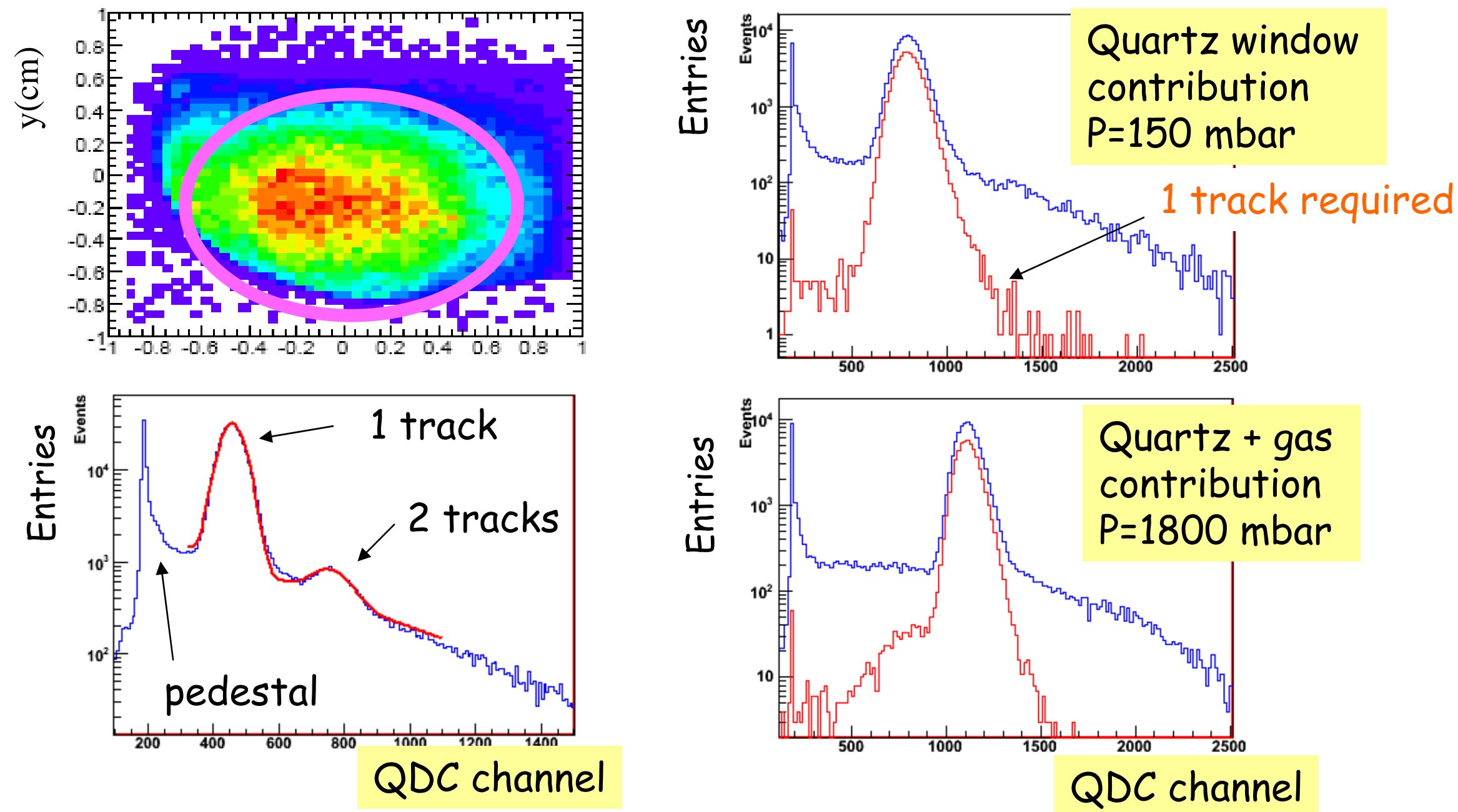


\section{Pressure scan}

$\checkmark$ Systematics tests on pressure and gas type

$$
\frac{n(E)^{2}-1}{n(E)^{2}+2}=\frac{\alpha P / T}{\sqrt{1-\left(E / E_{o}\right)^{2}}} \quad \theta_{c h}(E)=\frac{\sqrt{3 \alpha P / T}}{\left(1-\left(E / E_{o}\right)^{2}\right)^{1 / 4}} \quad \begin{gathered}
N_{c h} \propto P \\
\text { In the low } \mathrm{n} \text { limit }
\end{gathered}
$$

$\checkmark$ Reflections on the Al walls: $N_{R} \propto \theta_{c h} \propto \sqrt{P} \quad N_{p e} \propto N_{c h} R^{N_{R}} \propto P R^{\beta P}$

Al reflectivity

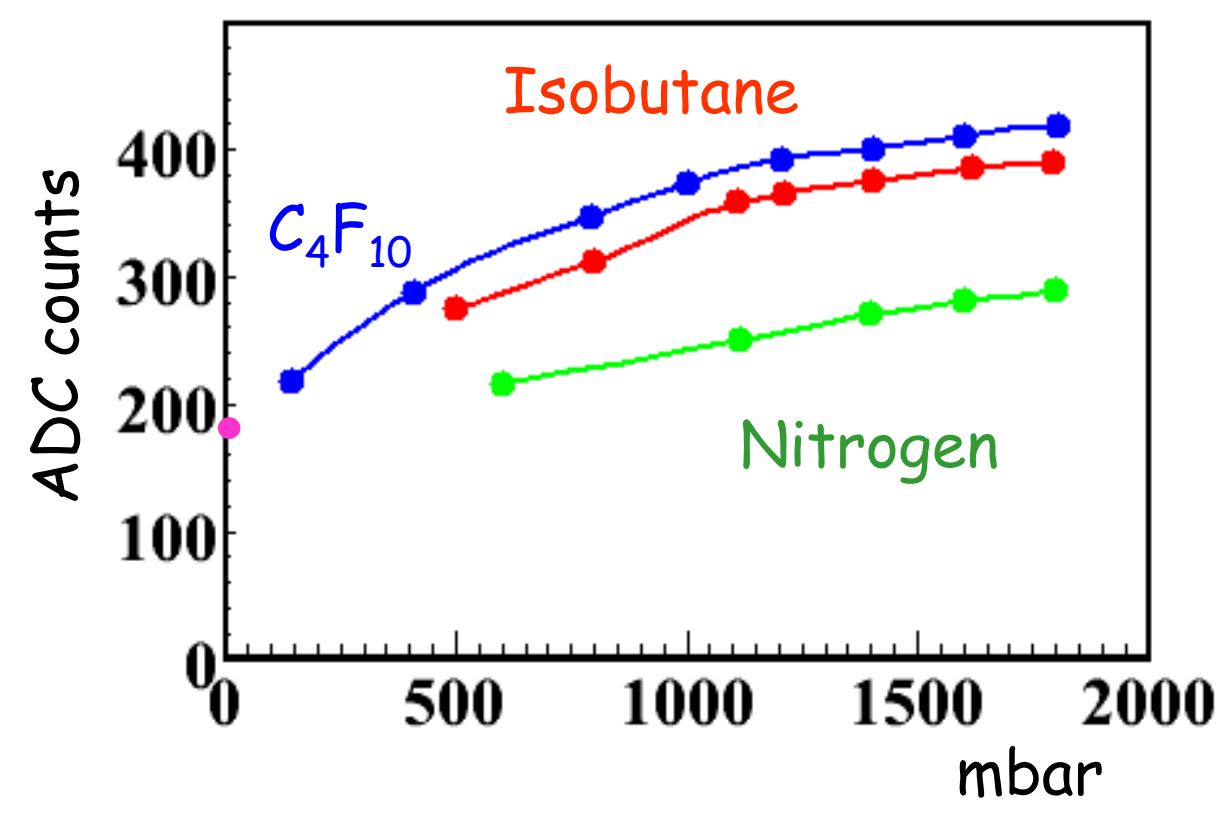

$\checkmark$ Al reflectivity measured against angles, wavelengths and polarization

$\checkmark$ Stringent tests for Monte Carlo (ongoing)

Mauro Villa - IEEE 09 - Orlando 


\section{LUCID history and present status}

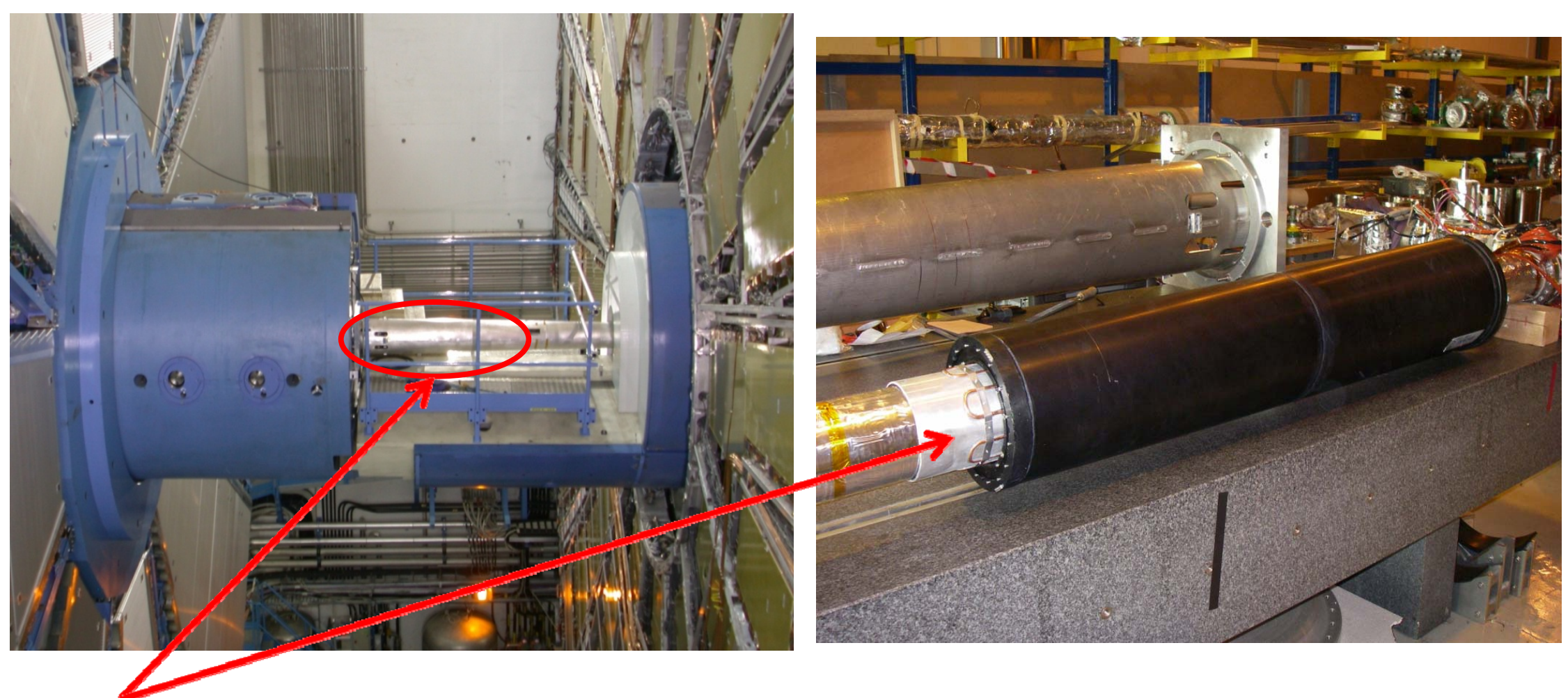

- LUCID detector construction was approved in ATLAS on February 2007

L LUCID is optimized to measure directly ATLAS luminosity during phase I of LHC (Luminosity $<4 \cdot 10^{33} \mathrm{~cm}^{-2} \mathrm{~s}^{-1}$ )

$\square$ The detector was ready on summer 2008 to catch the first LHC beam splash events

$\square$ Although some new implementations/maintenances are still ongoing, the detector is in advanced phase of commissioning 


\section{Trigger rates during the first $L H C$ beam parus}
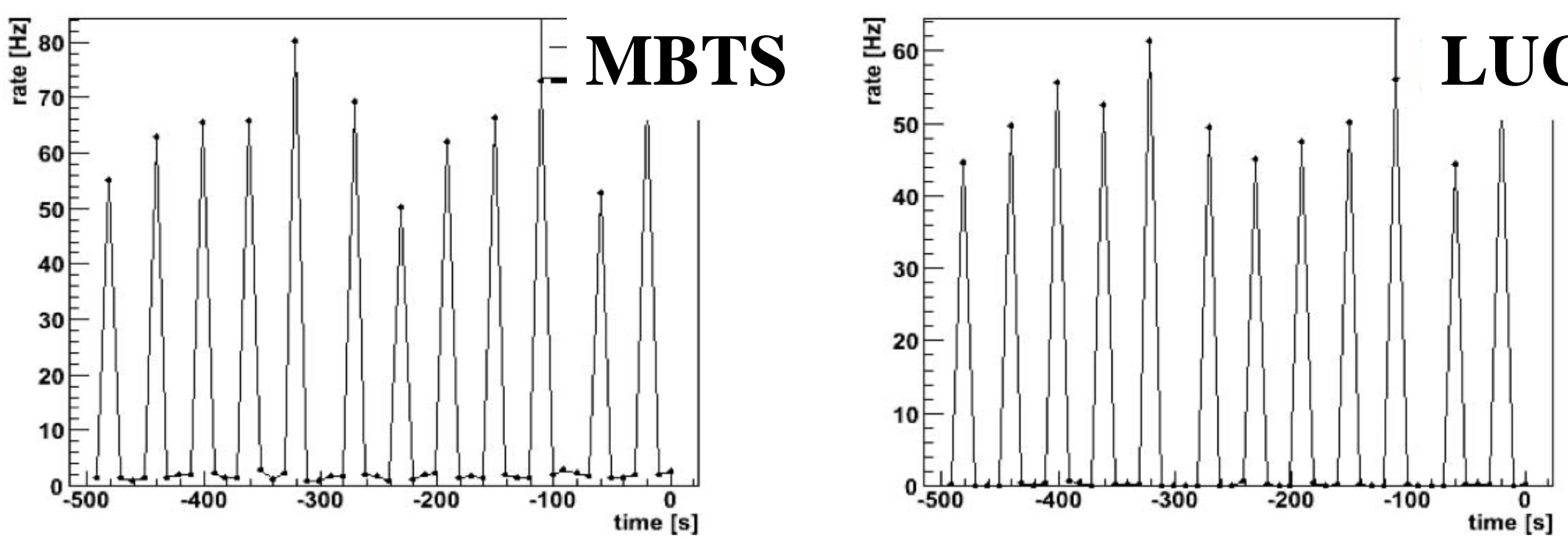

$\square$ MBTS - Minimum Bias Trigger Scintillators: Array 16+16 scintillators, placed symmetrically to the IP, covering $2.1<\eta<3.8$

$\square$ LUCID - At least 1 hit in one of the two arms within $5.6<\eta<5.9$

LUCID will provide one of the MB trigger to ATLAS 


\section{Conclusions}

$\checkmark$ The LUCID detector is designed to provide ATLAS with:

- Luminosity monitor on-line/off-line

- Luminosity for each Bunch Crossing and Integrated

- Minimum Bias Trigger

- Beam Control monitor

$\checkmark$ LUCID is a running detector in advanced status of commissioning

$\checkmark$ The luminosity will be measured using a combination of many methods

$\checkmark$ A beam test has been performed to check tube performances in several experimental conditions $\rightarrow$ systematics used to tune the MC. Analisys ongoing. 


\section{Back up slides}
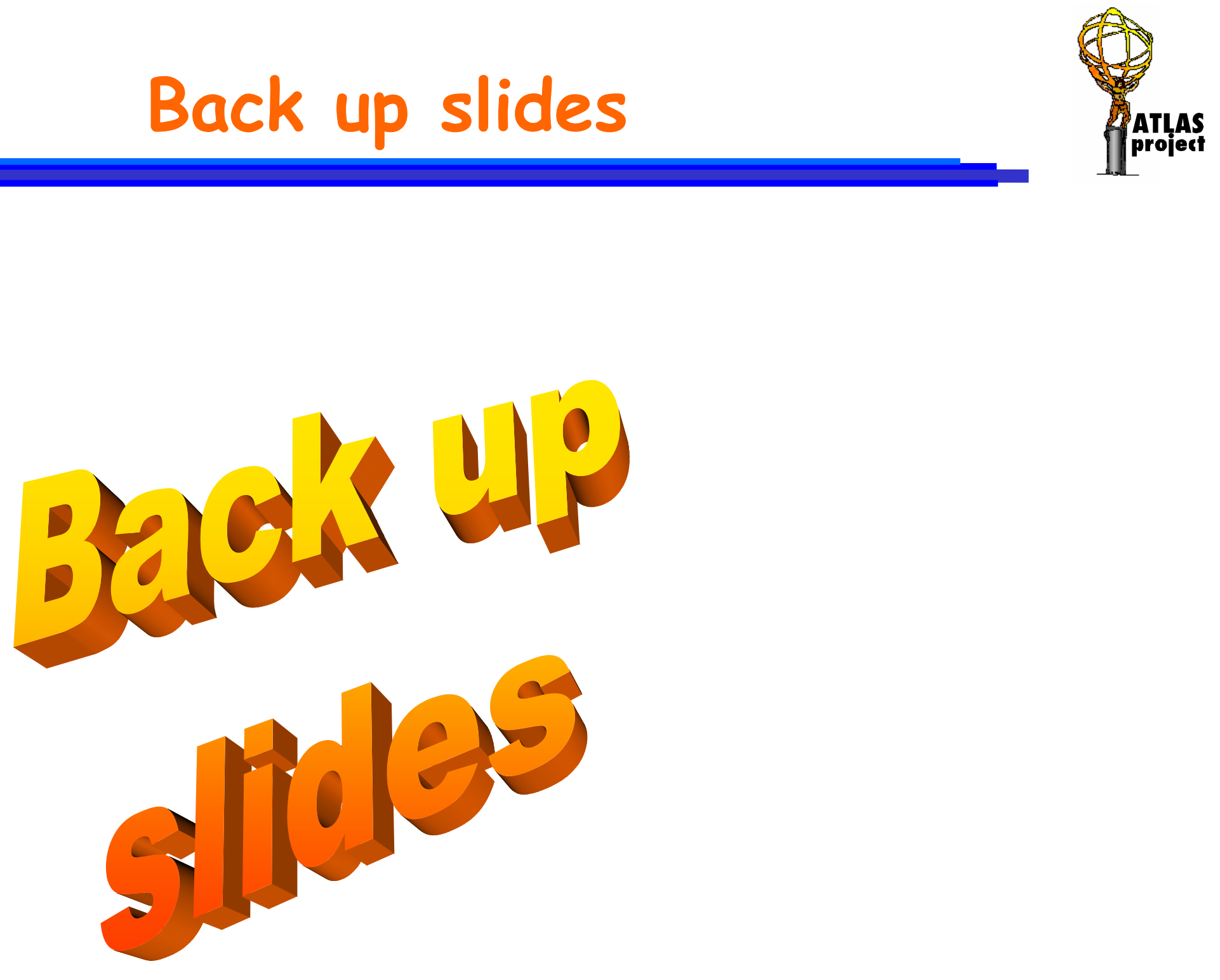

Mauro Villa - IEEE 09 - Orlando 


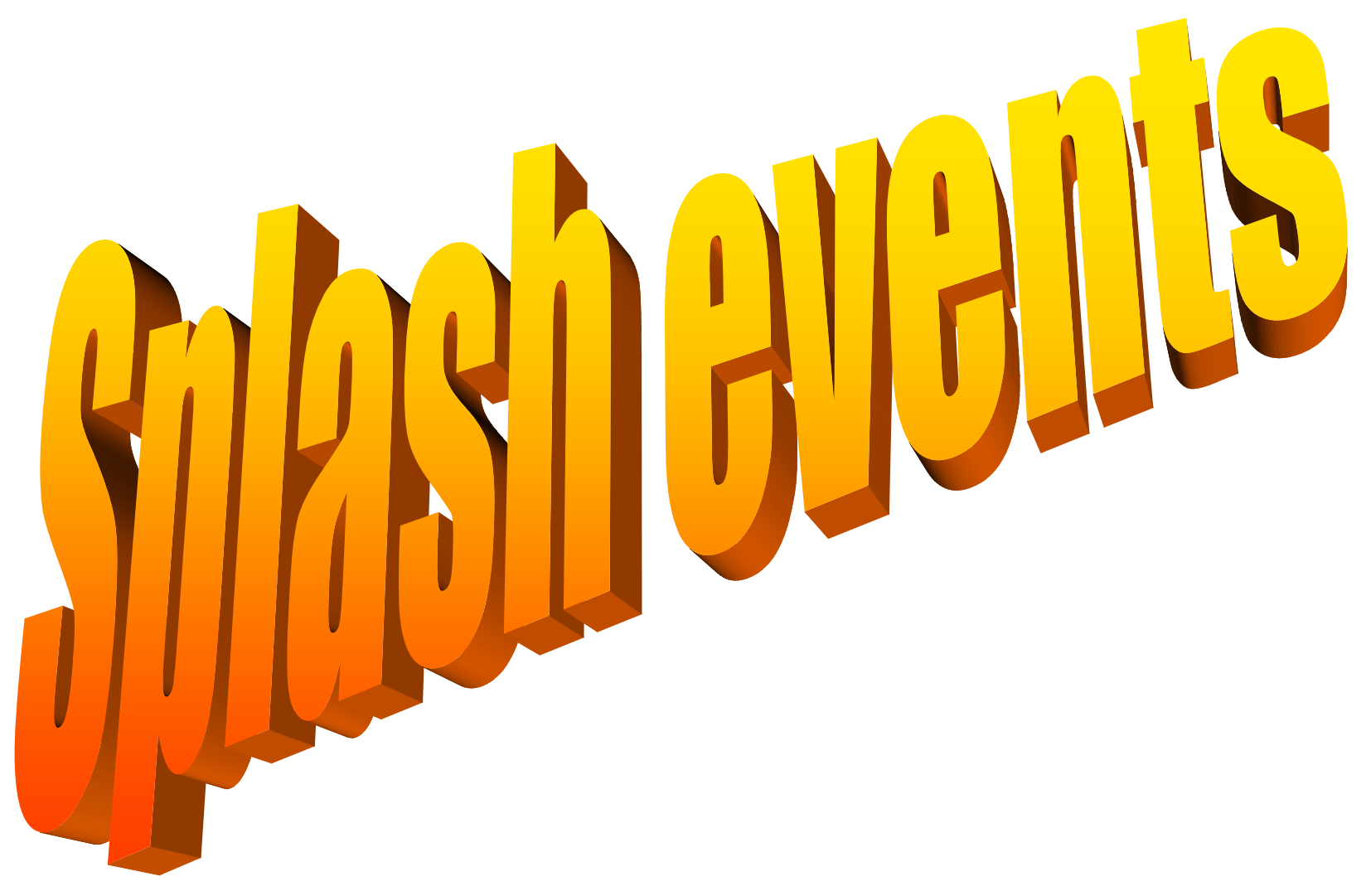

Mauro Villa - IEEE 09 - Orlando 


\section{First HITS seen at LHC startup}

C A

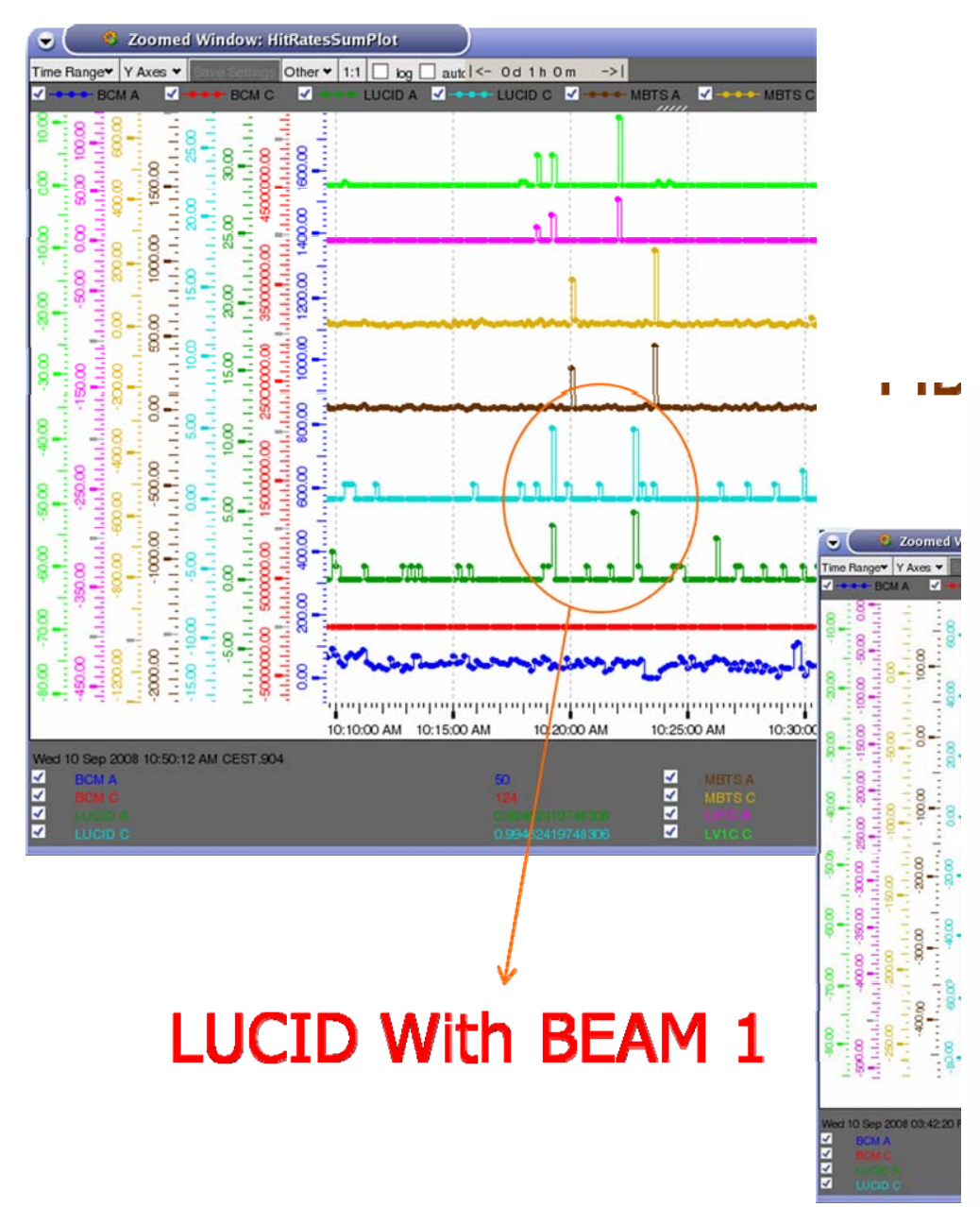
side side

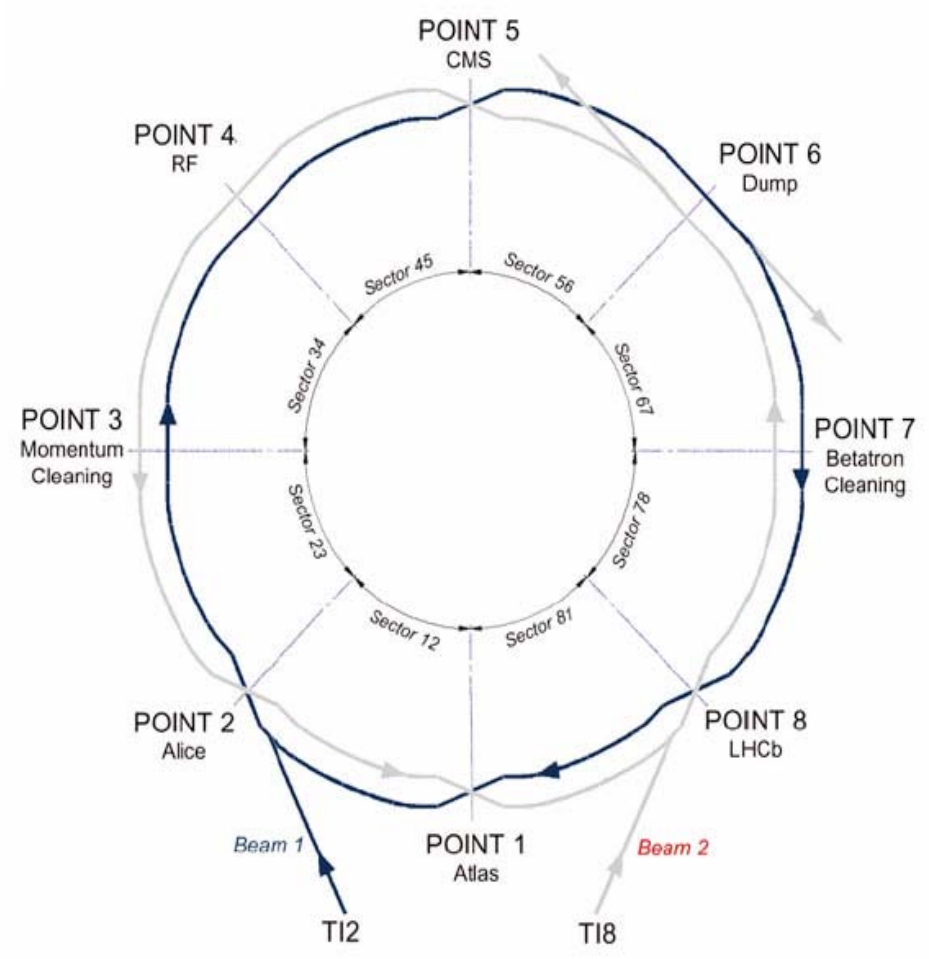

$\square$ LUCI D recorded the very first LHC “splash" events from the two beams 


\section{September $10^{\text {th }}$ events with Beam 2}
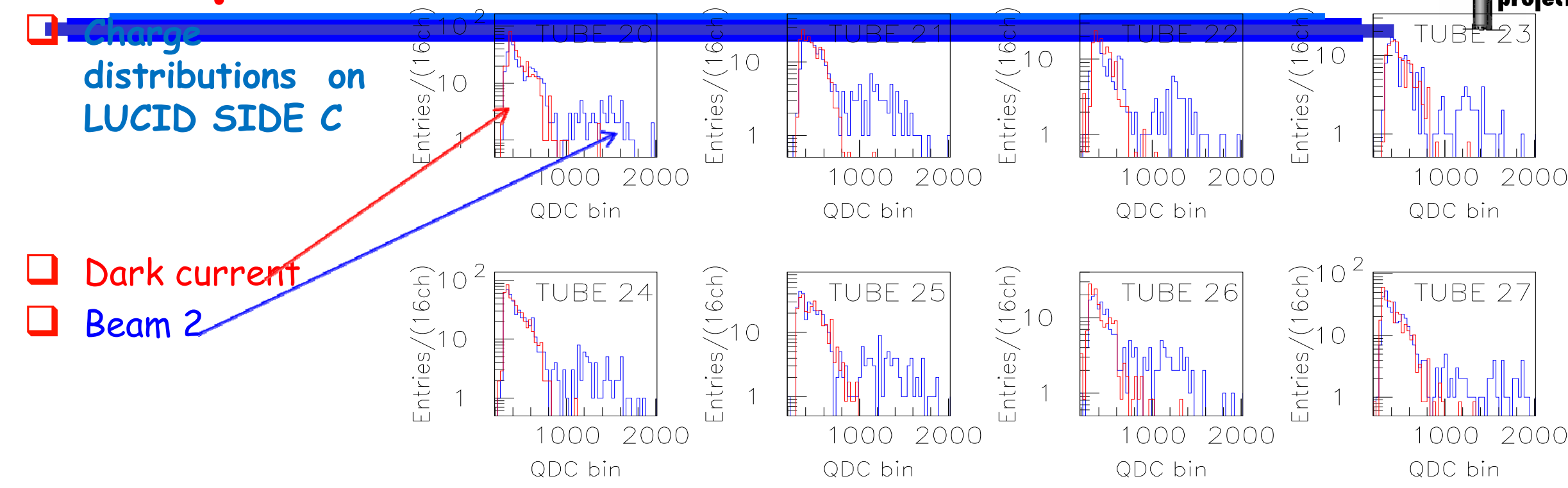

fatlas project
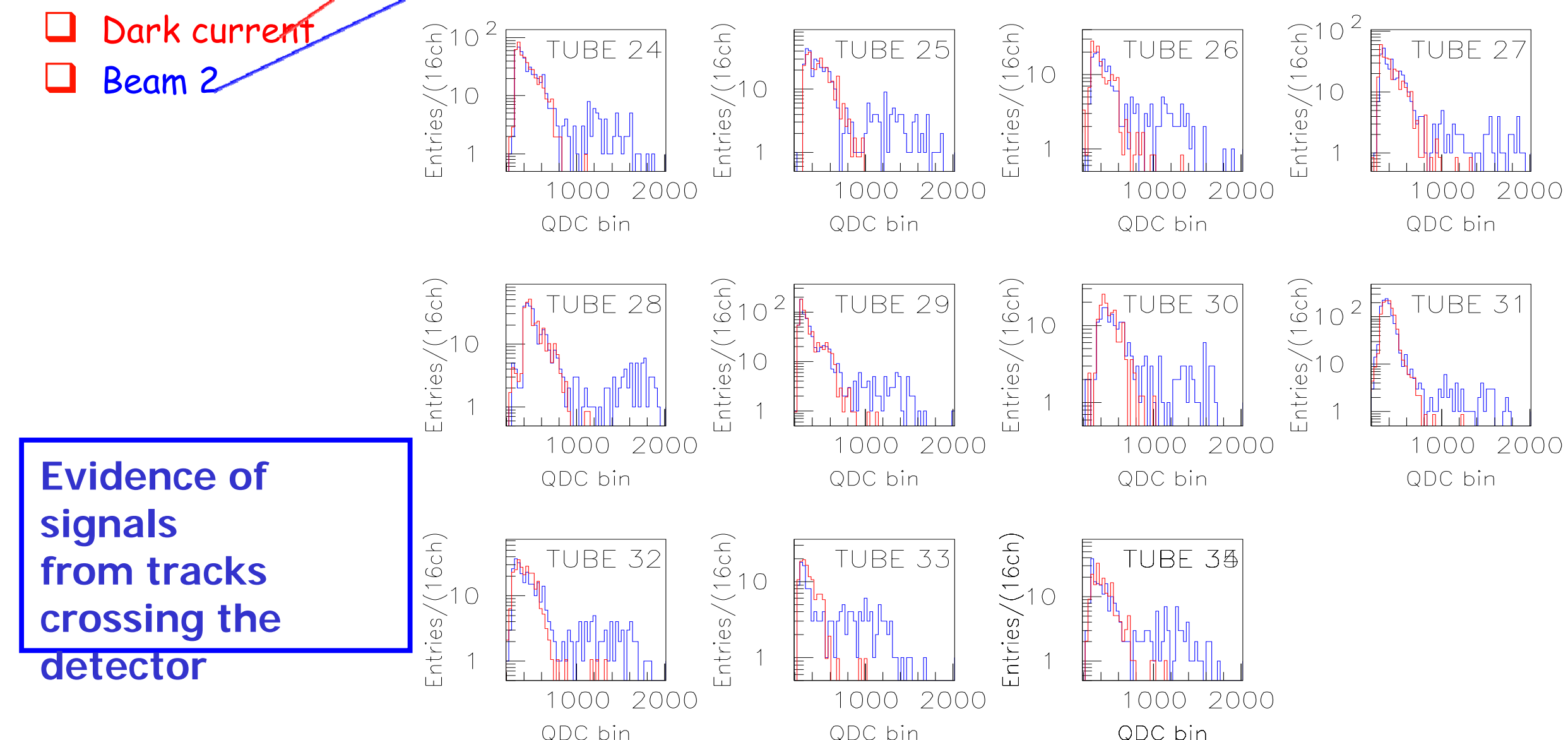

QDC bin

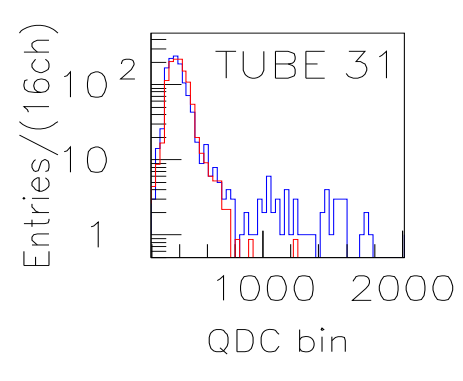

Mauro Villa - IEEE 09 - Orlando 


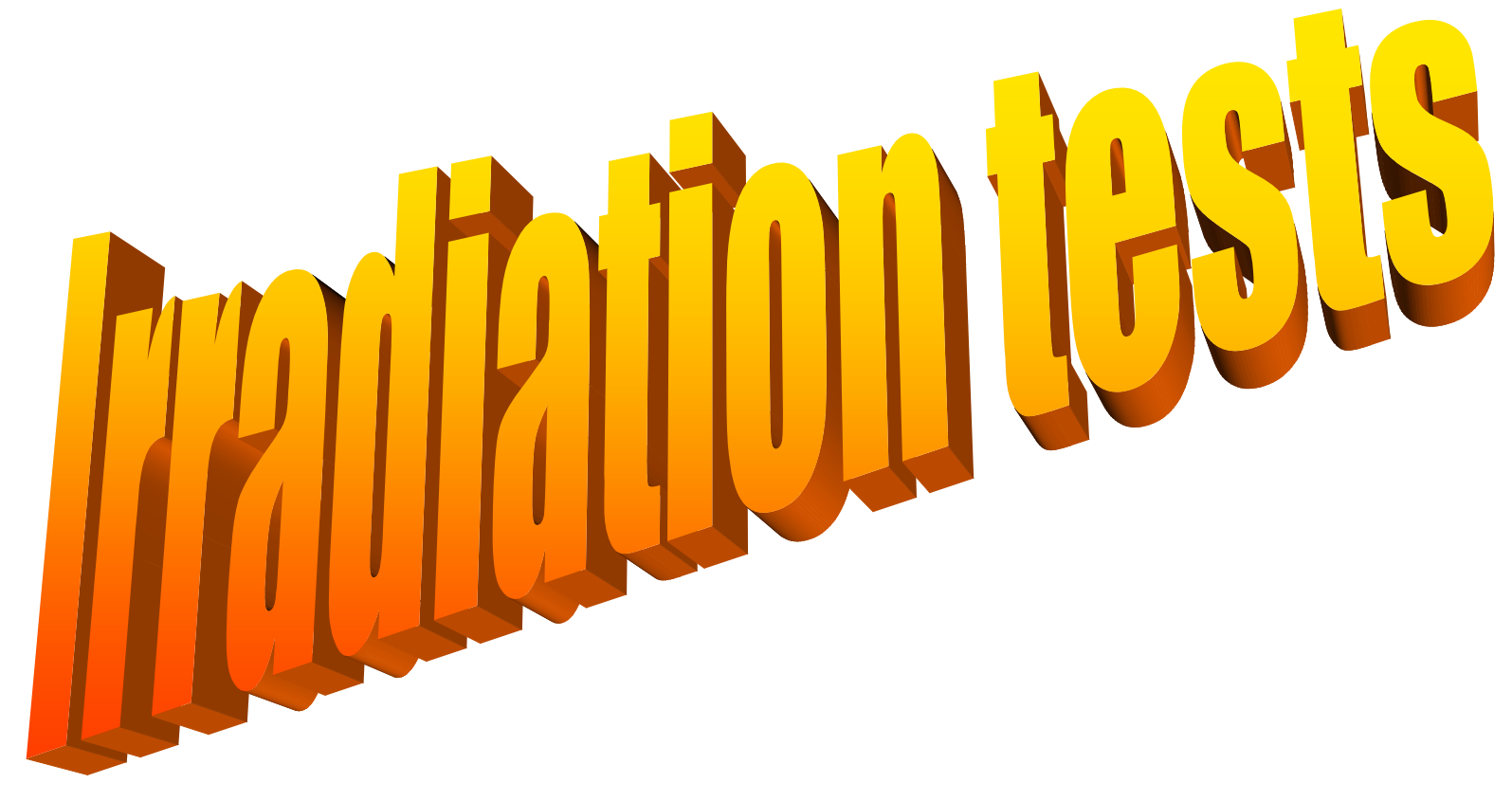

Mauro Villa - IEEE 09 - Orlando 


\section{Gamma irradiation tests}
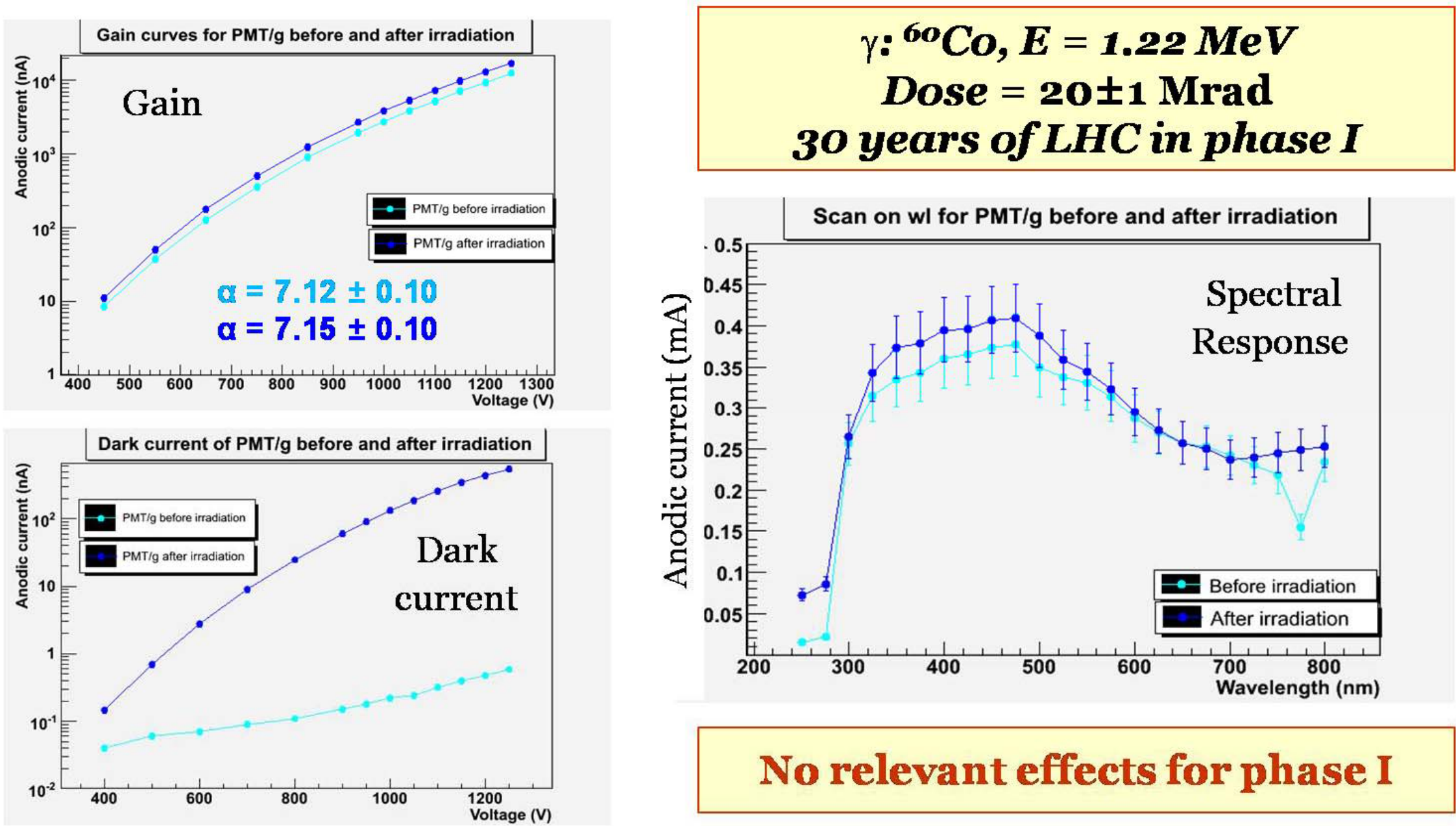

Mauro Villa - IEEE 09 - Orlando 


\section{Neutron irradiation test}

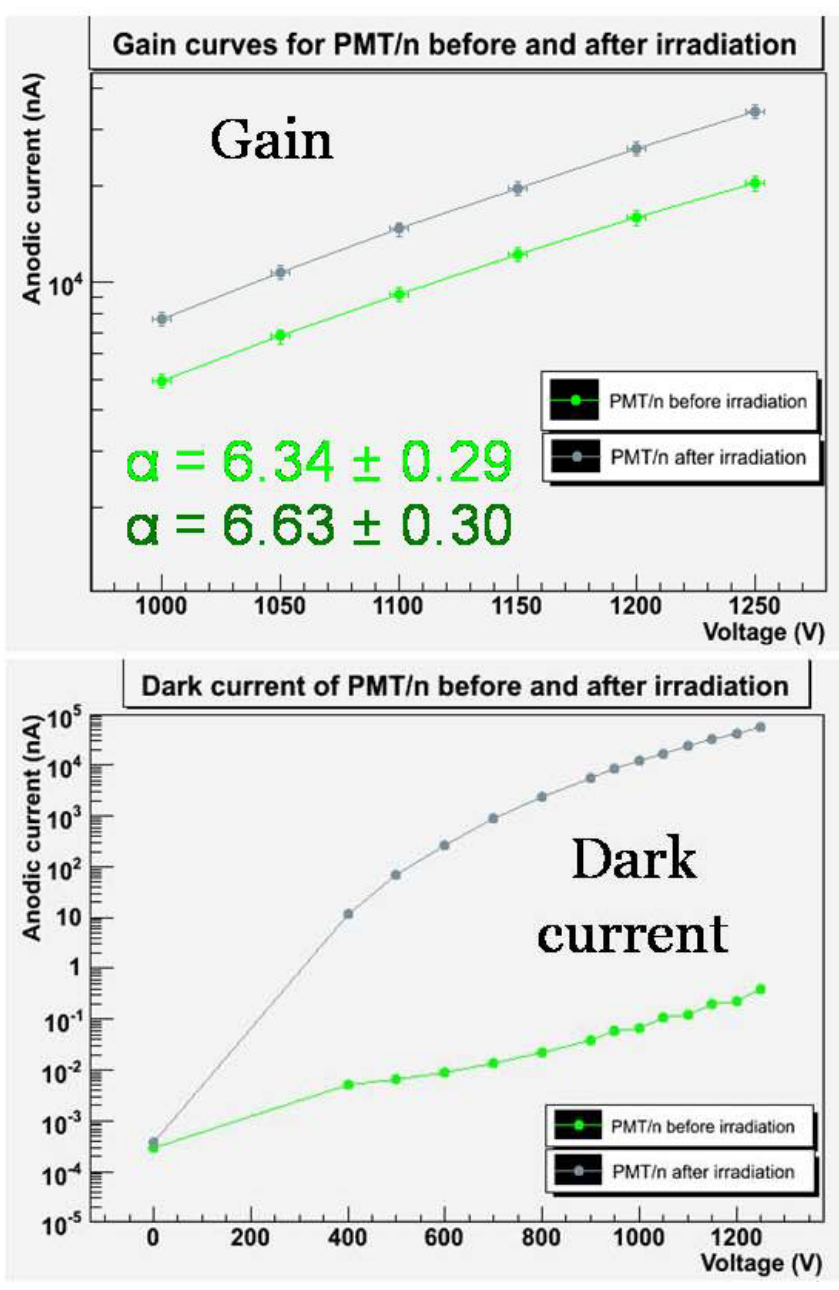

\section{n: ENEA-Casaccia reactor $E=10 O \mathrm{KeV}$ \\ Dose $=10$ years of LHC in phase I}

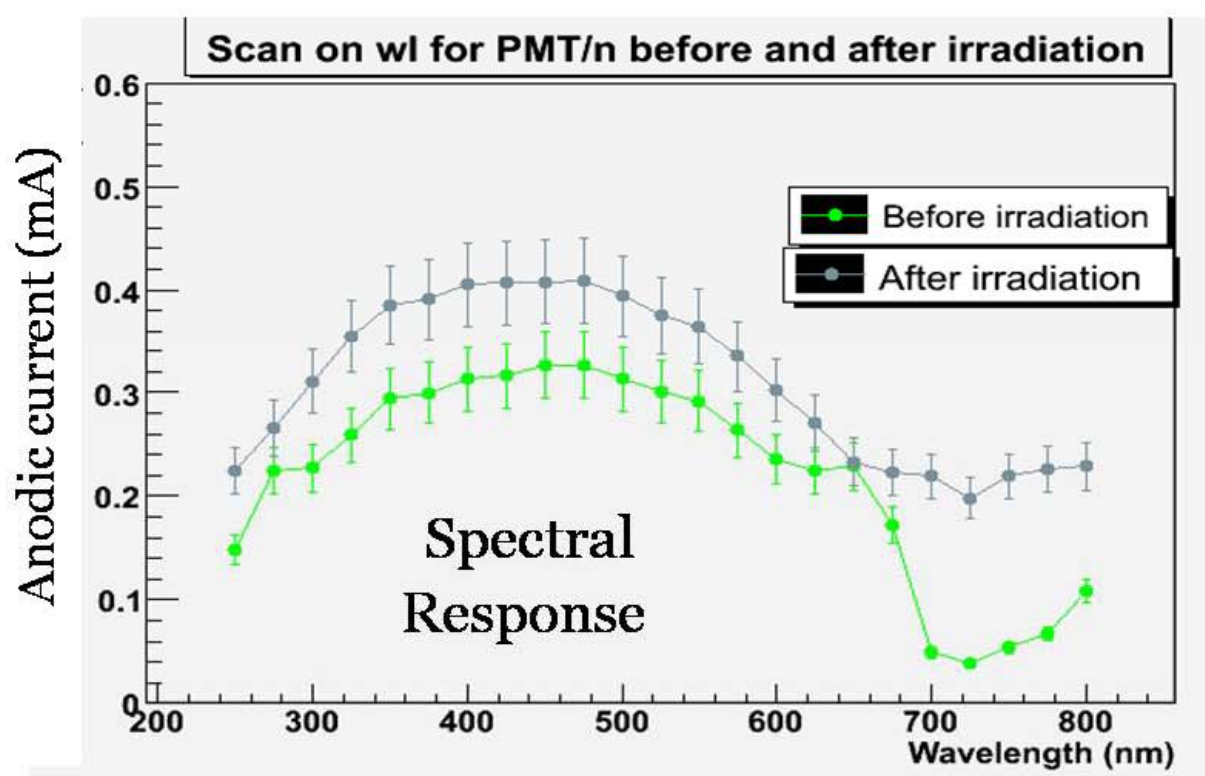

No relevant effects for phase I 


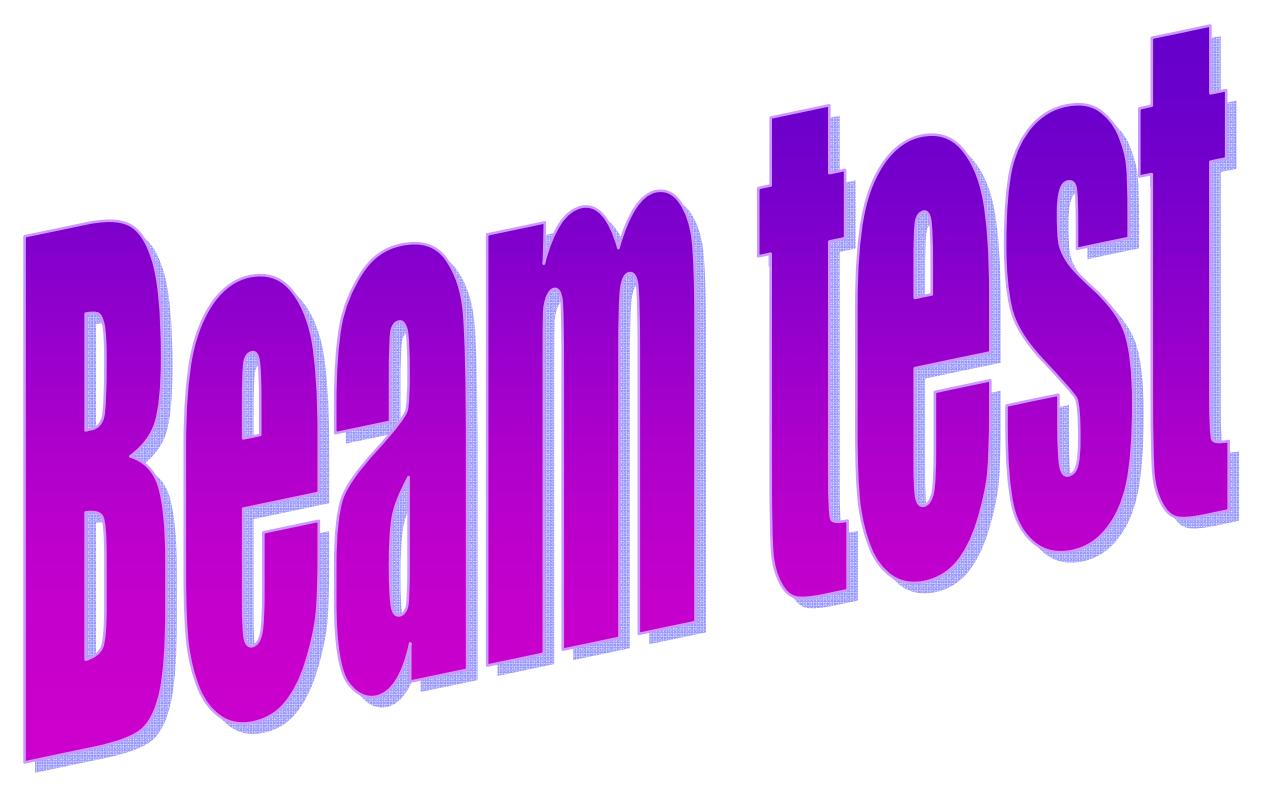

Mauro Villa - IEEE 09 - Orlando 
Set up del Detector

\section{LUCID con 6 tubi}

- 3 come quelli installati in Atlas

- 1 non lucidato internamente

- 1 rivestito internamente di mylar CERN RAV

- 1 mecchanically polished

DESY $M$

DESY $M$

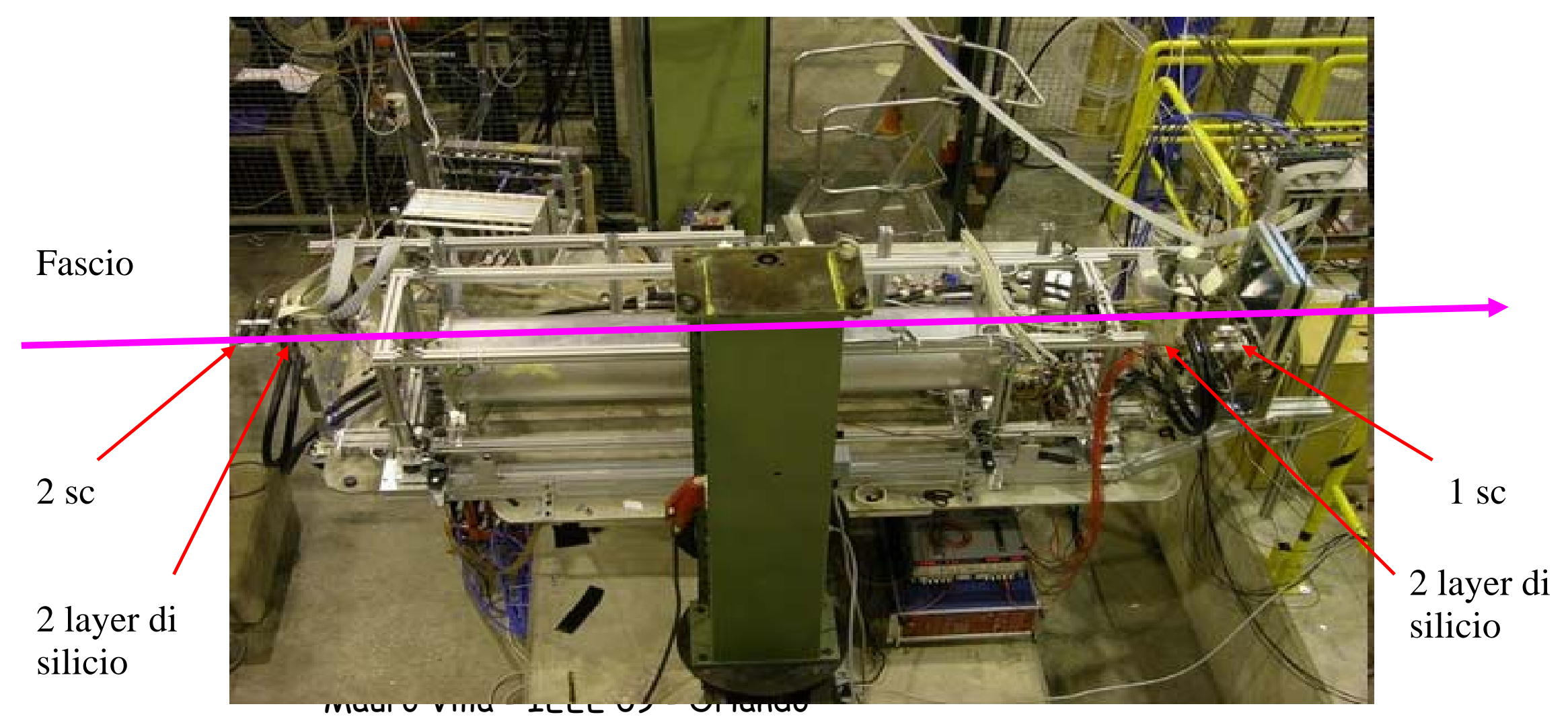


silicio entro $0.5 \mathrm{~mm}$ della regione aspettata

Precisione dell'estrapolazione delle tracce nel LUCID 20 $\mu \mathrm{m}$
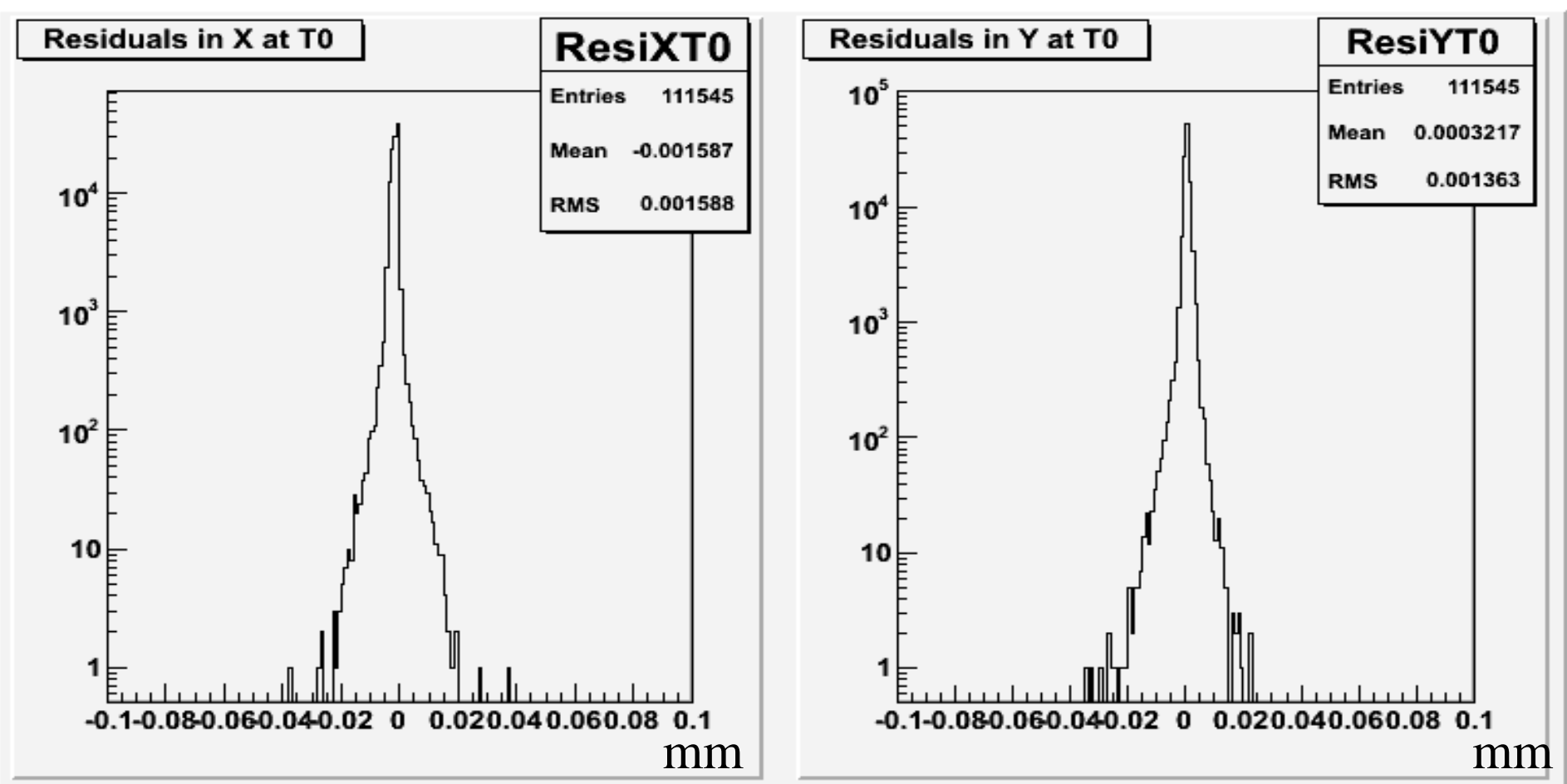

- Tracce ricostruite e fittate nei 4 strati di silicio

- Metodo dei residui:

coordinata fittata nel primo strato di silicio - coordinata ricostruita inforioni su allineamento e risoluzione (ok per scopi LUCID)

Mauro Villa - IEEE 09 - Orlando 


\section{CONDIZIONI DEL FASCIO}

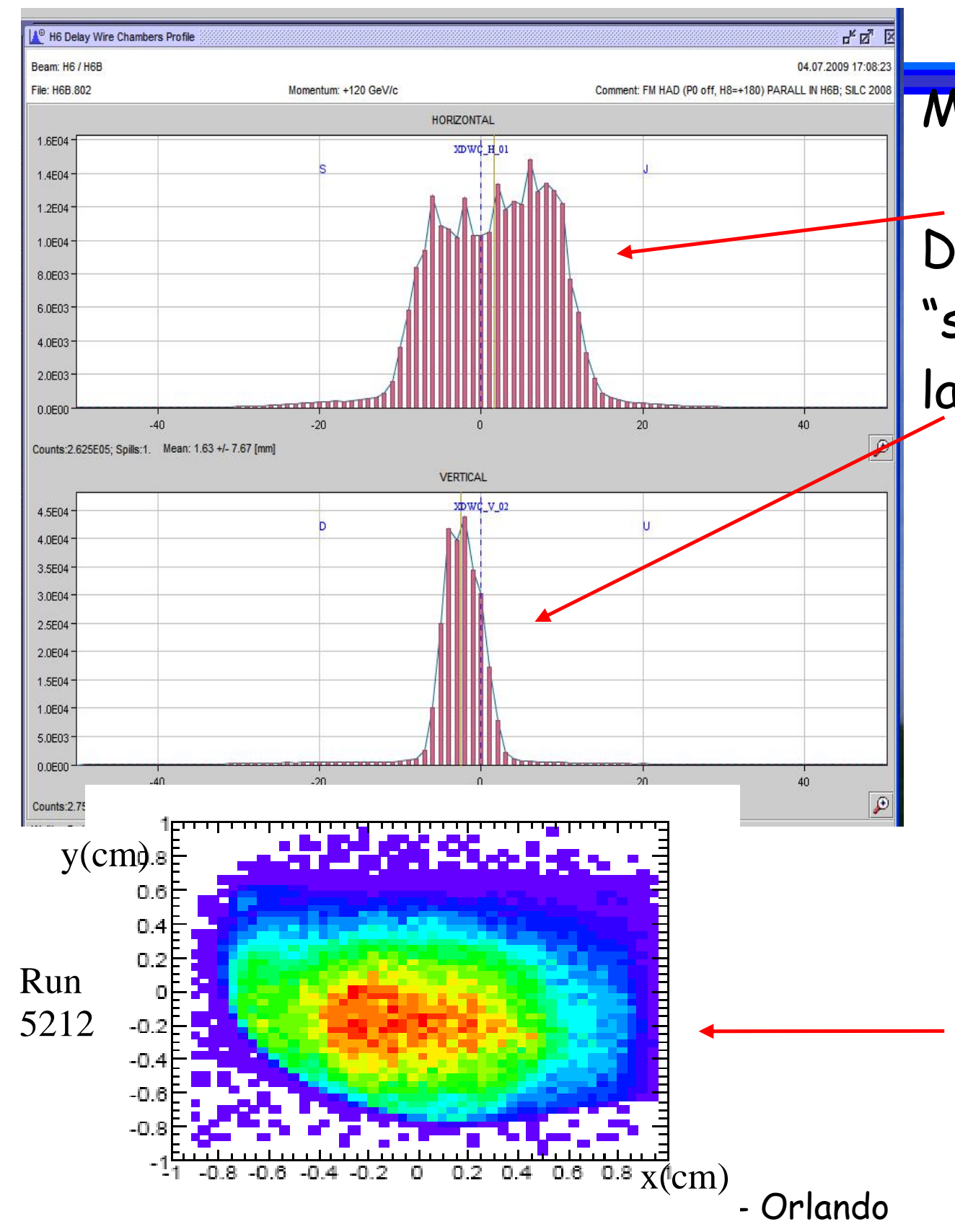

Fascio visto dai layer di silicio

$\stackrel{m}{3} 5 \times 10^{11}$ protoni per bunch dal SPS

$\sim 2.2 \times 10^{5}$ pioni positivi da $120 \mathrm{GeV}$ sulla linea Bturteh di 9s ogni 30s 


\section{SCAN IN PRESSIONE}
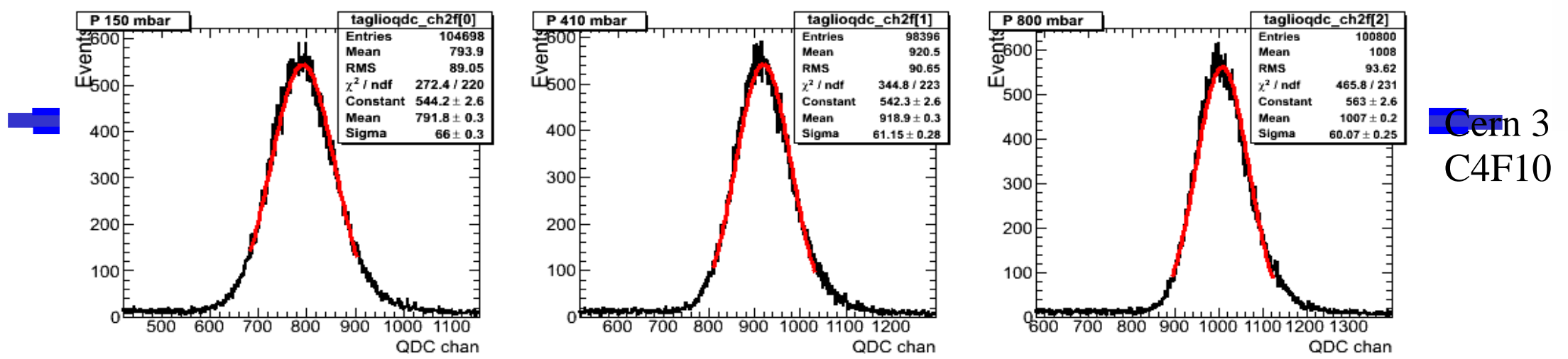

ATLAS
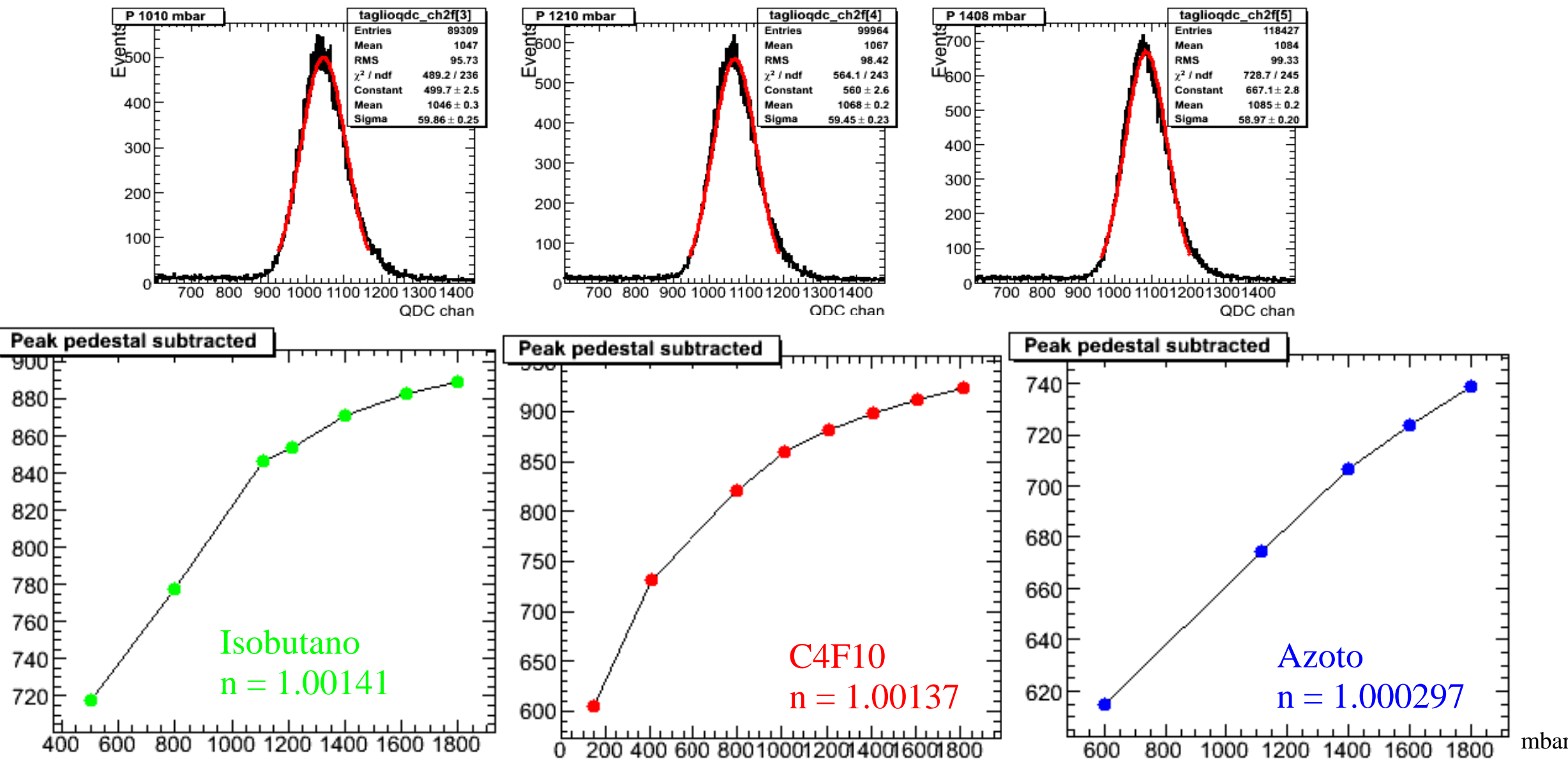
SCAN ANGOLARE
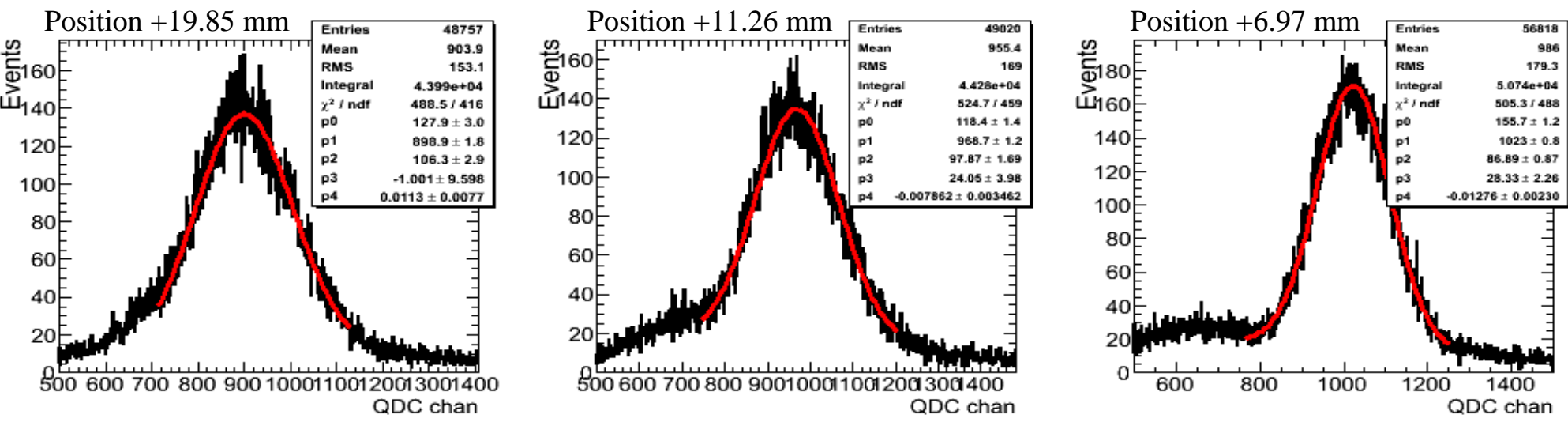

PATLAS QDC chan

QDC chan
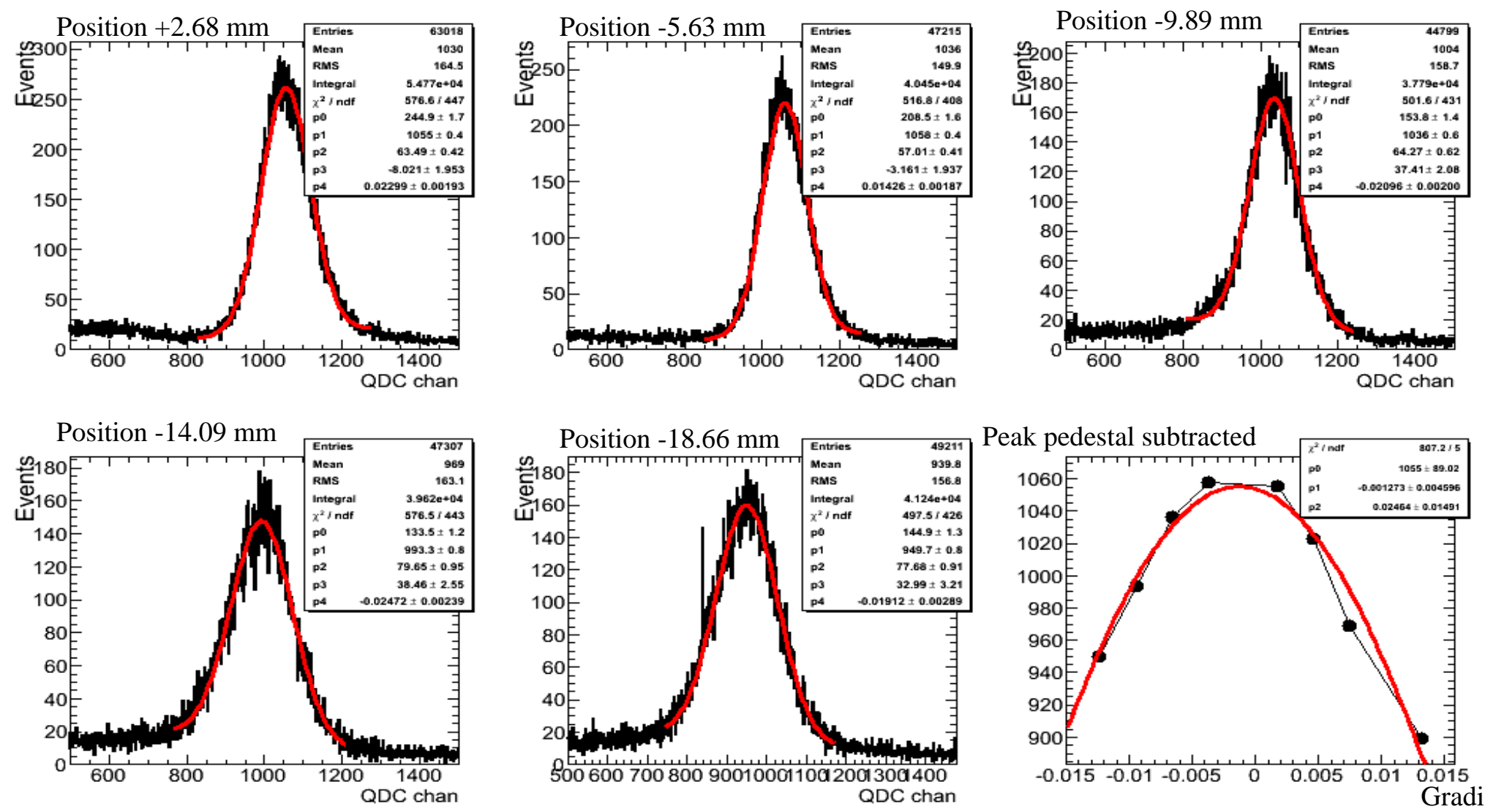


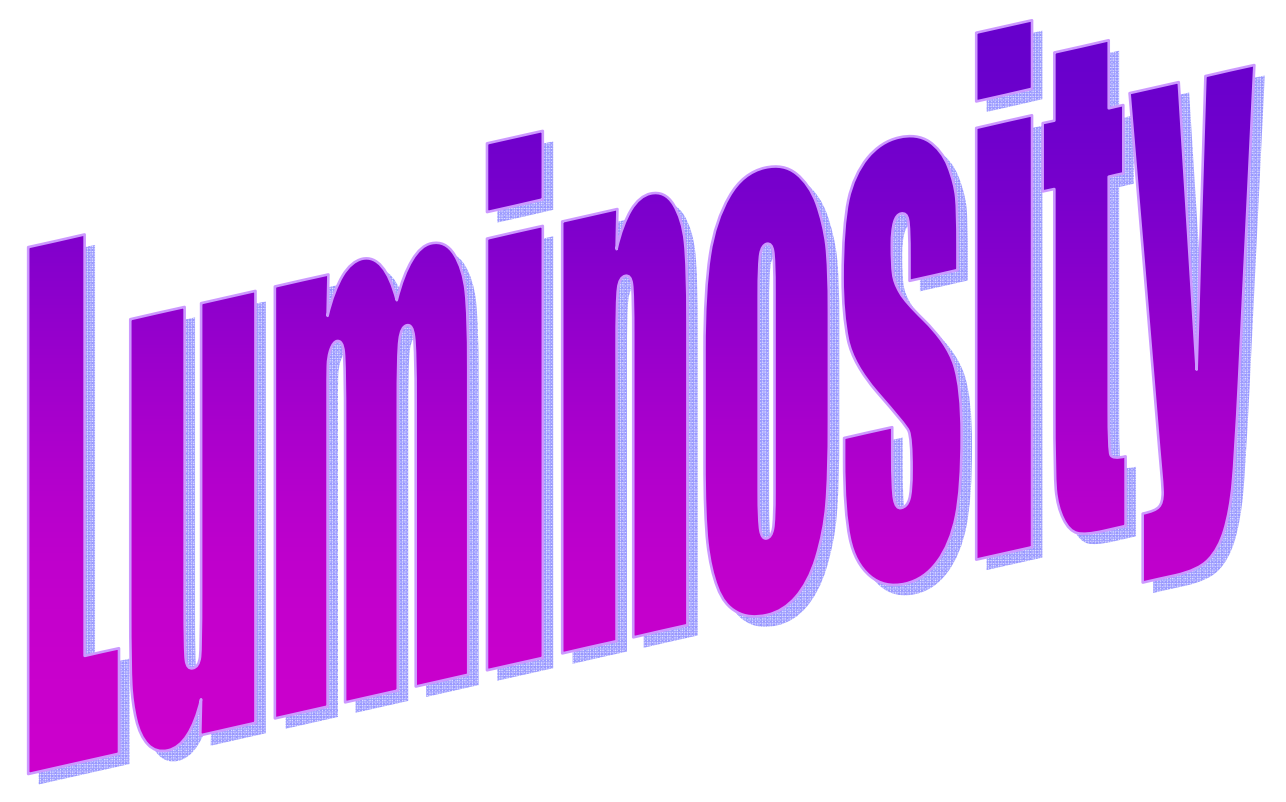

Mauro Villa - IEEE 09 - Orlando 


\section{Physics Interest in L}

$\checkmark$ Absolute Luminosity:

$\checkmark$ measure cross sections for standard physics

$\checkmark$ measure Higgs production cross section

$\checkmark$ observe deviations from SM and New Physics

$\checkmark$ Requirements:

$\checkmark$ ultimate precision at the 2-3\% level

$\checkmark$ different methods needed for cross check

$\checkmark$ minimize systematics

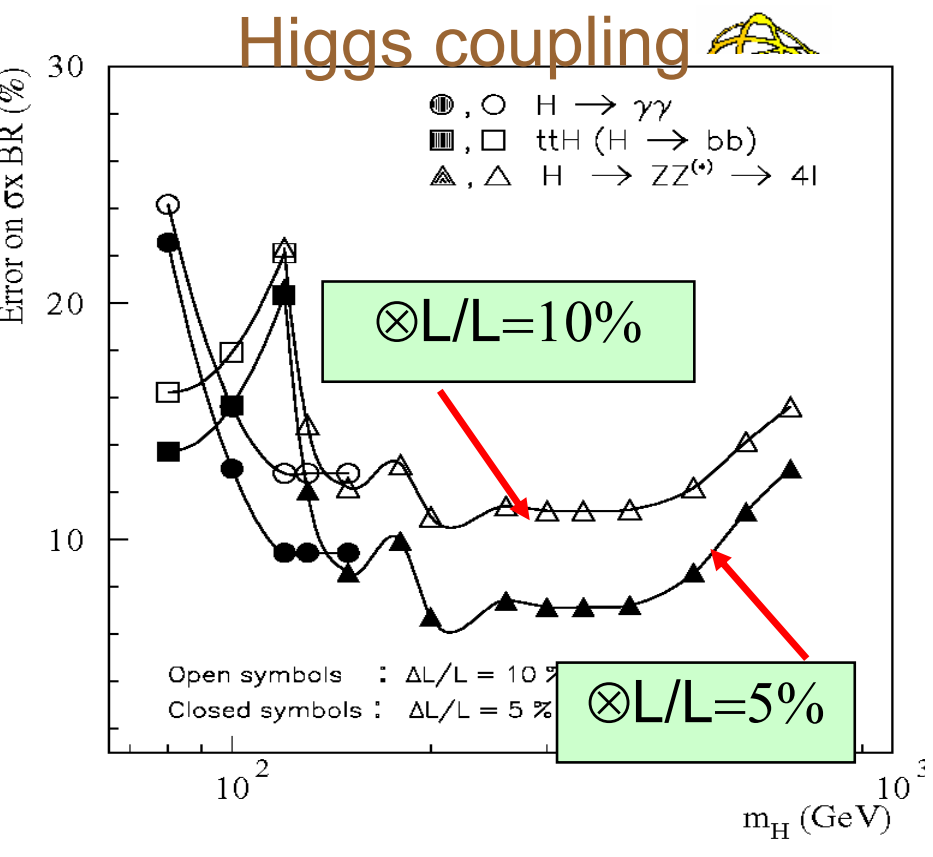

Systematic error due to luminosity (ATLAS TDR )

\section{COMBINED EFFOR'T FROM DIFFERENT DETECTORS Beam structure}

$\checkmark$ Relative luminosity:

$\checkmark$ beam stability

$\checkmark$ beam degradation (efficient use of trigger)

$\checkmark$ evaluate trigger \& DAQ dead-times

$\checkmark$ determine beam background

$\checkmark$ Luminosity Block spread

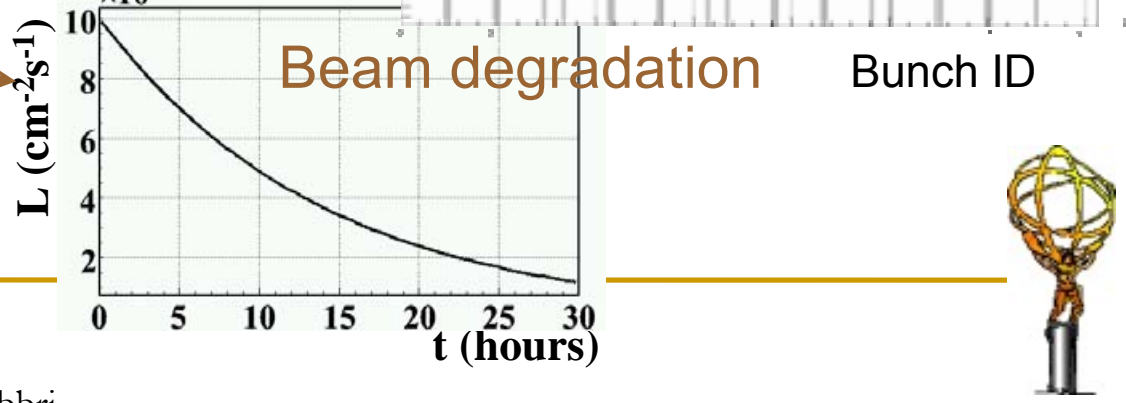




\section{Luminosity from LHC parameters}

$$
L=\frac{f \sum_{i=1}^{k_{b}} N_{1 i} N_{2 i}}{\text { Impact surface }}=\frac{f \sum_{i=1}^{k_{b}} N_{1 i} N_{2 i}}{4 \pi \sigma_{x}^{*} \sigma_{y}^{*}}=\frac{f k_{b} N^{2}}{4 \pi \varepsilon_{N} \frac{\beta^{*}}{\gamma}}
$$

$N_{x i}=$ number of protons in bunch $i$ of beam $x ; f=$ revolution frequency; $\sigma_{x}, \sigma_{y}=$ transverse beam dimensions at the IP; $k_{b}=$ number of bunches; $\beta^{*}=\beta$ function at IP; $\varepsilon_{N}=\sigma_{x}^{*} \sigma_{y}^{*} / / \beta^{*}$ normalized emittance; $\gamma=E / m_{p}(\sim 7460)$

\section{Accuracy limited by}

- Extrapolation of $\sigma_{x} \sigma_{y}$ from measurement point to IP

- Precision in measurement of bunch currents

- Beam-beam effects at IP, beam crossing angle, ... Maximum precision obtainable from machine $5-10 \%$ 


\section{Goal precision on $\mathrm{L} \sim 2-3 \%$}

*Elastic scattering in Coulomb-Nuclear Interference region to get $L$ and $\sigma_{\text {tot }}$ at $\mathrm{L} \sim 10^{27} \mathrm{~cm}^{-2} \mathrm{~s}^{-1}$ -optical theorem as a back-up solution ALFA detector in Roman Pots

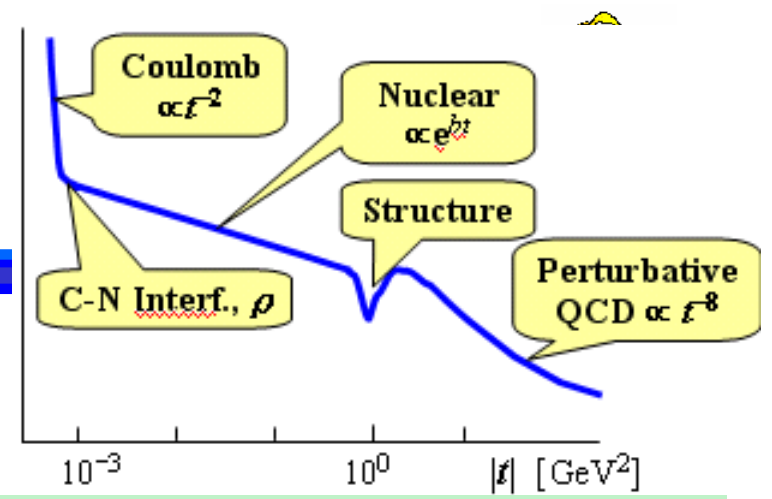

$$
\left.\frac{d N}{d t}\right|_{t=C N I}=L \pi\left|f_{C}+f_{N}\right|^{2} \approx L \pi\left|-\frac{2 \alpha_{\mathrm{EM}}}{|\mathrm{t}|}+\frac{\sigma_{\mathrm{tol}}}{4 \pi}(i+\rho) e^{-\frac{b t}{2}||^{2}}\right|^{2}
$$

*Luminosity monitor calibrated at low lumi but working up to $\mathrm{L} \sim 10^{34} \mathrm{~cm}^{-2} \mathrm{~s}^{-1}$ LUCID

$$
\sigma_{\text {tot }}=\frac{16 \pi}{1+\rho^{2}} \times \frac{\left.(d N / d t)\right|_{t=0}}{N_{e l}+N_{\text {inel }}}
$$

$$
\mu_{L U C I D}=\frac{\sigma_{p p} g \varepsilon_{L U C I D} g L}{f_{B X}}
$$

:Absolute L from QED (pp $\rightarrow \mathrm{pp} \mu \mu)$ and QCD

$$
R_{X}=\sigma_{X} g L
$$

$(\mathrm{W} \rightarrow|\mathrm{v}, \mathrm{Z} \rightarrow| \mathrm{l})$ processes (need to control PDF)

.Improve Luminosity from machine with ZDC

*Further luminosity/beam monitoring with BCM, MBTS... 


\section{ATLAS Forward Detectors}
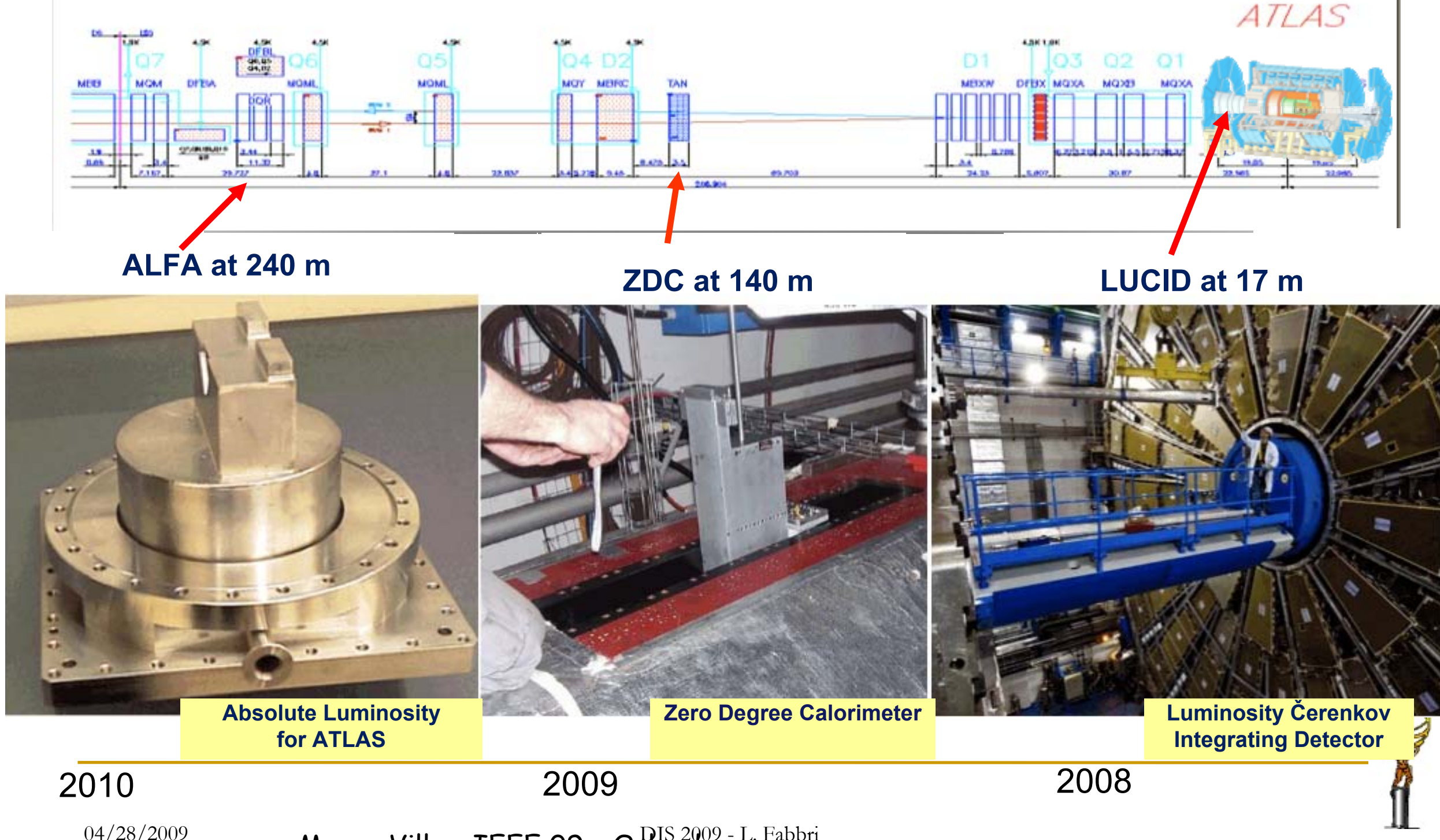

04/28/2009

Mauro Villa - IEEE 09 - Orlando 
5 board piggy back

$\checkmark$ Main FPGA

$\checkmark$ (Stratix

$\checkmark 2$ EPMC

$\checkmark$ (cyclone II)

$\checkmark$ S-link

$\checkmark$ TTCrq

$$
\text { (1) }
$$

PMTs

(CFD)

Performance:

$40 \mathrm{Mhz}$ bus clocks

8 Gbit input rate

1 Gbit output rate

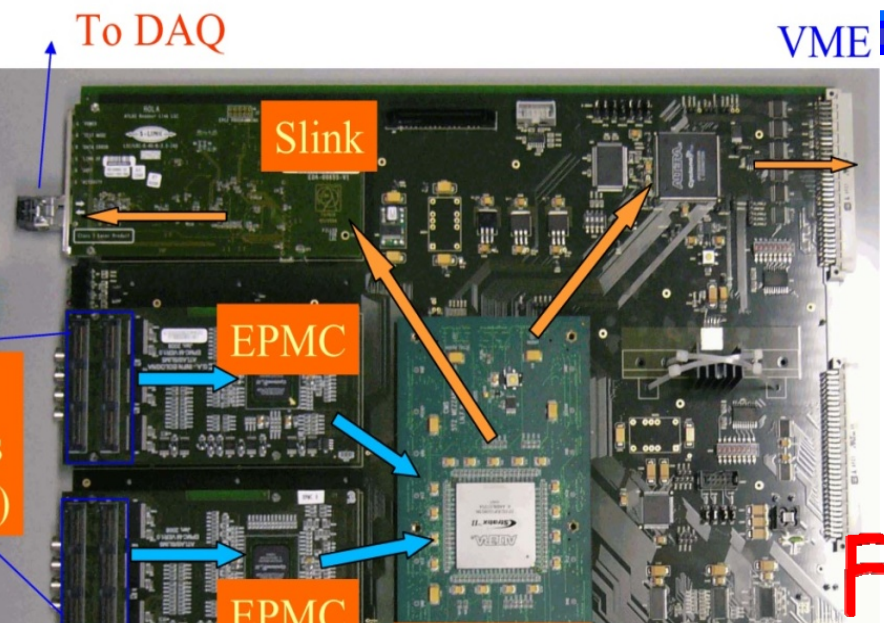

And Trigger card

$\mathrm{VME}$

Final goal: online monitor

$\checkmark$ Luminosity by BC

$\checkmark$ Flexibility on Iuminosity and trigger algorithms

$\checkmark$ Lucid hits in the ATLAS data stream

Mauro Villa - IEEE 09

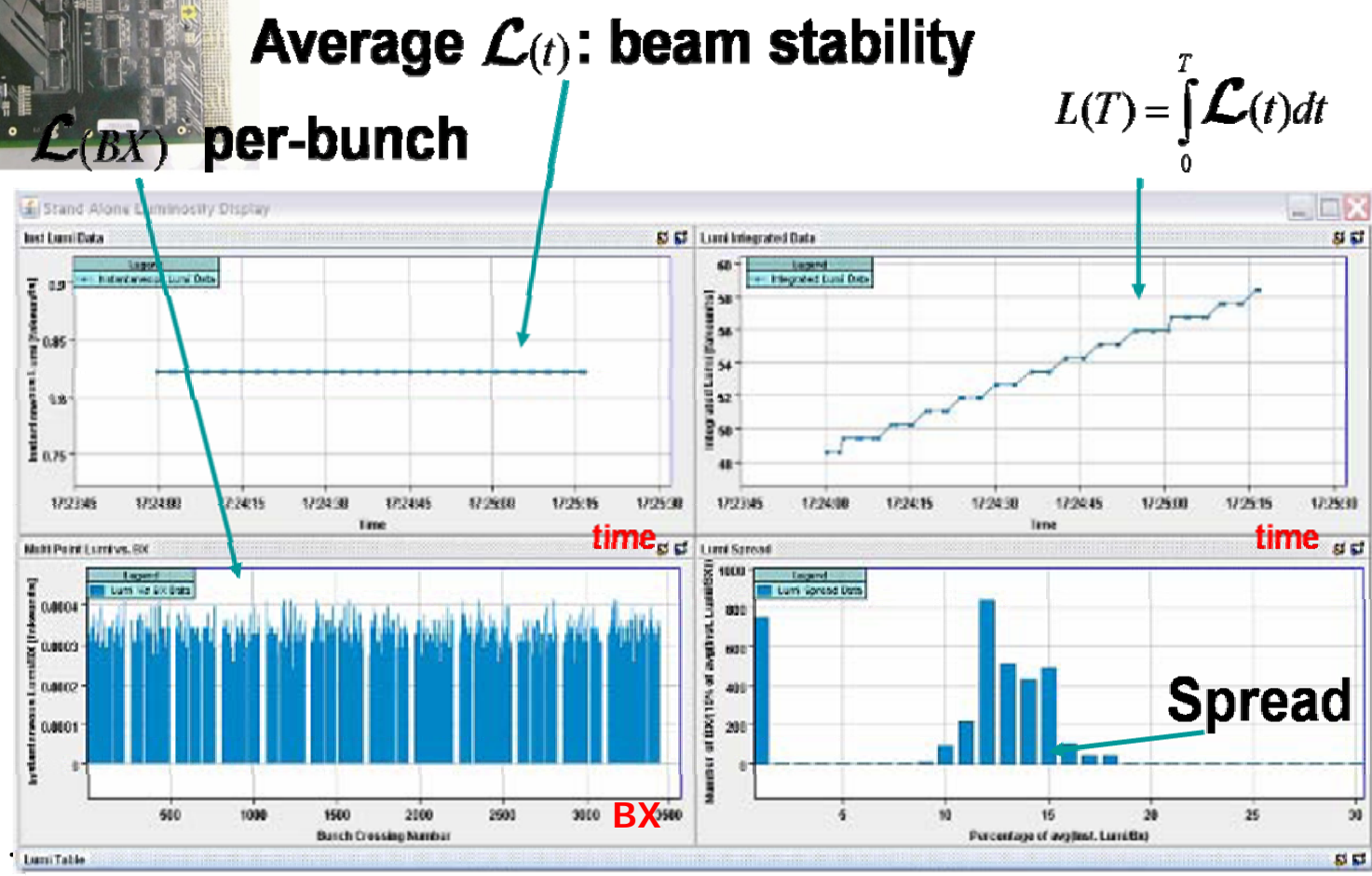




\section{Luminosity}

$$
L_{B X}=\frac{\mu_{B X}}{\sigma_{p p}^{i n e l}}=\frac{L}{f_{B X}}
$$

$$
f_{B X}=\frac{n_{B X}}{3564} \times 40 M H z
$$

\begin{tabular}{|c|c|}
\hline$L_{B X}\left[\mathrm{~cm}^{-2}\right]$ & Average bunch luminosity \\
\hline$\mu_{B X}$ & Mean number of inelastic proton-proton interactions per bunch crossing \\
\hline$\sigma_{p p}{ }^{\text {inel }}\left[\mathrm{cm}^{2}\right]$ & Inelastic proton-proton cross section (MB + SD + DD) \\
\hline$L\left[\mathrm{~cm}^{-2}, \mathrm{~s}^{-1}\right]$ & Instantaneous luminosity \\
\hline$f_{B X}\left[\mathrm{~s}^{-1}\right]$ & Bunch crossing frequency \\
\hline
\end{tabular}

- $\sigma_{\mathrm{pp}}^{\text {inel }}=79.2 \mathrm{mb}$ (PYTHIA6.2)

- $\sigma_{\mathrm{pp}}^{\text {inel }}=84.5 \mathrm{mb}$ (PHOJ ET1.12)

- Luminosity ranges from $10^{27}$ (calibration) to $10^{34}$ (LHC design)

- At design luminosity, $\mathrm{n}_{\mathrm{BX}}=2808 \rightarrow \mu_{\mathrm{BX}} \sim$ 25 


\section{LUCID calibration}

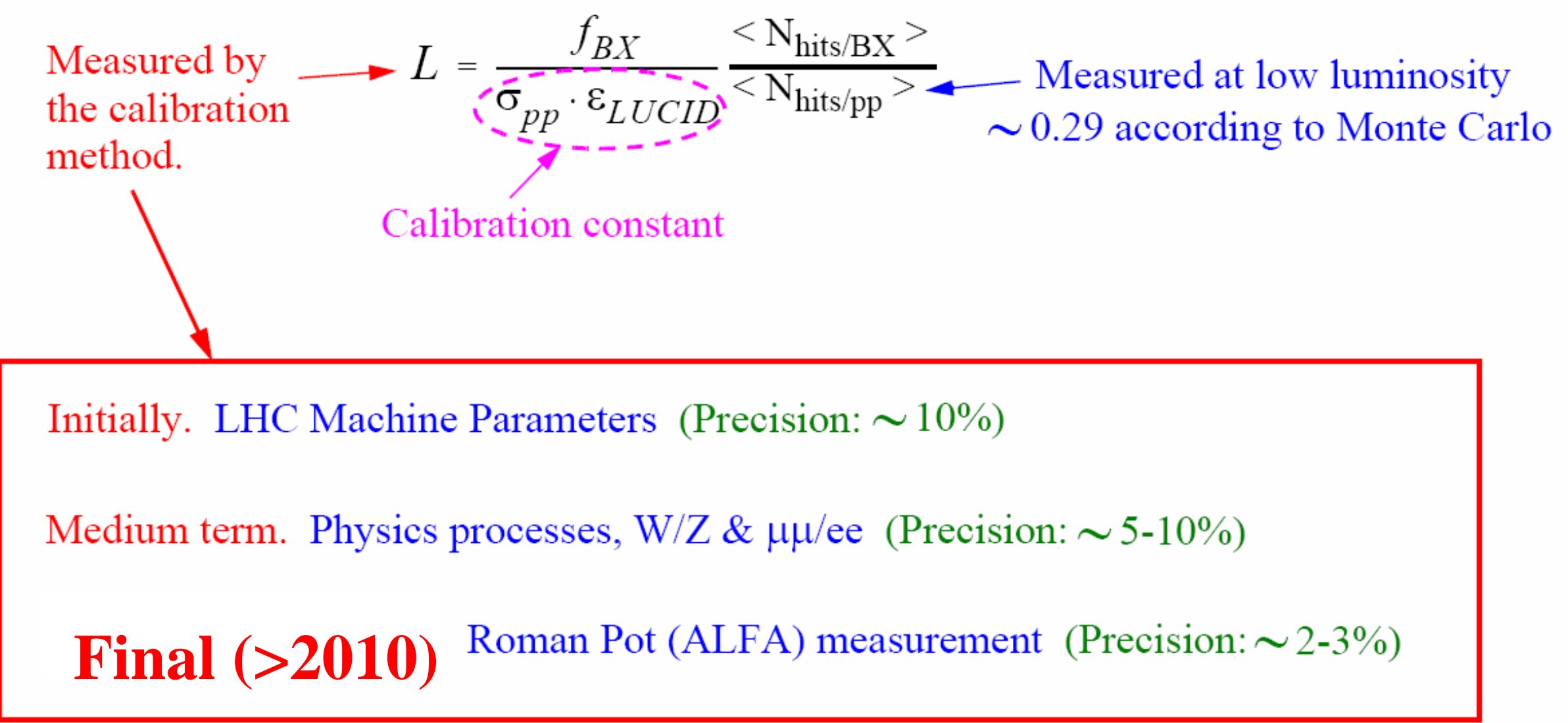

Mauro Villa - IEEE 09 - Orlando 


\section{Luminosity monitoring}

$\checkmark$ Average number of tracks per tube $\Xi^{\mathrm{E}}$ per event proportional to luminosity.

$\checkmark$ Monitor bunch by bunch stability. Measure relative luminosity

$\checkmark$ Calibration needed:

$\checkmark$ LHC machine parameters

$\checkmark$ Know reactions e.g. Z,W

$\checkmark$ ALFA calibration in special runs

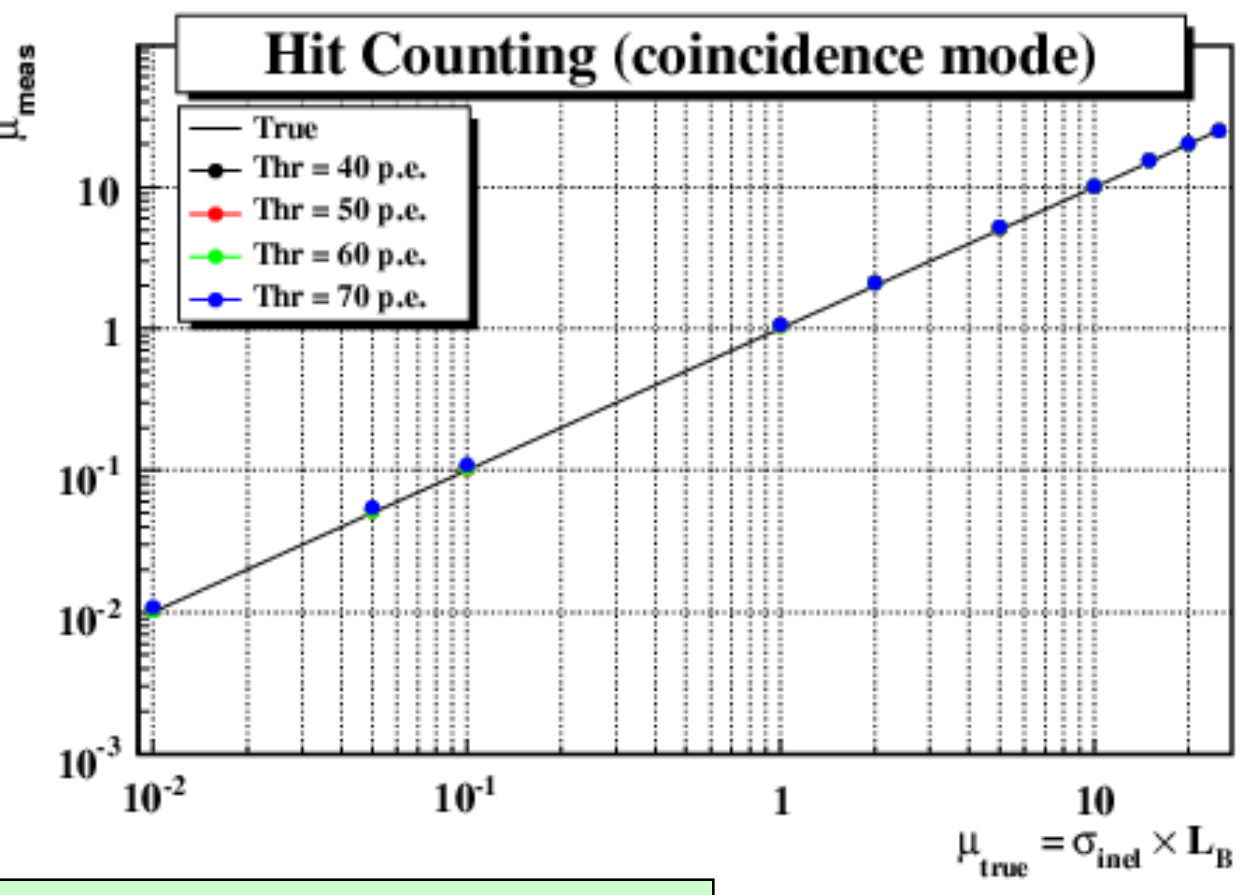

$$
\mu_{M E A S}=\frac{\langle M\rangle}{\langle N\rangle \cdot \varepsilon}=L \cdot \sigma_{\text {inel }} \longrightarrow L=\frac{\langle M\rangle}{\langle N\rangle \cdot \varepsilon \cdot \sigma_{\text {inel }}}
$$

$\mu=$ average number of interactions per bunch crossing

$<M>=$ average number of charged particles per bunch crossing

$<N>=$ average number of particles per interaction $\varepsilon=$ interaction efficiency

$\sigma_{\text {inel }}=$ inelastic cross sec. 


\section{Calibration}

$L_{B X}=\frac{\mu_{B X}}{\sigma_{p p}^{i n e l}}=\frac{\langle M\rangle}{\sigma_{p p}^{\text {inel }} \times \varepsilon_{p p} \times\langle C\rangle}=k_{L U C I D} \times\langle M\rangle$

$\mathrm{k}_{\mathrm{LUCID}}$ is the LUCID

calibration constant

Calibration with Monte Carlo

$k_{L U C I D}=\frac{1}{\sigma_{p p}^{\text {inel }} \times \varepsilon_{p p} \times\langle C\rangle} \quad \varepsilon_{\mathrm{pp}}$ and $<\mathrm{C}>$ are extracted from single pp interaction events

Calibration with Data at low luminosity $\left(\mu_{\mathrm{BX}}<<1\right)$

$k_{L U C I D}=\frac{L_{B X}^{A L F A, L H C}}{\langle M\rangle} \quad \begin{aligned} & <\mathrm{M}>\text { is measured by LUCID } \\ & \mathrm{L}_{\mathrm{BX}} \text { is measured by LHC or ALFA }\end{aligned}$

$<\mathrm{C}>$ can also be measured by LUCID at low luminosity and cross-checked with MC

$12 / 11 / 2008$

A. Sbrizzi

Mauro Villa - IEEE 09 - Orlando 


\section{Summary of sys uncertainties}

\begin{tabular}{|l|c|c|c|c|}
\hline & Mode & Calibration & Range & Systematics \\
\hline Zero counting & Single side & Monte Carlo & $\mu<2$ & $1 \%$ \\
\hline Zero counting & Coincidence & Monte Carlo & $\mu<2$ & $2 \%$ \\
\hline Hit counting & Single side & Monte Carlo & $\mu<2$ & $2.6 \%$ \\
\hline Hit counting & Coincidence & Monte Carlo & $\mu<2$ & $6 \%$ \\
\hline Hit counting & Single side & Data & any $\mu$ & $3 \%$ \\
\hline Hit counting & Coincidence & Data & any $\mu$ & $4 \%$ \\
\hline
\end{tabular}

Mauro Villa - IEEE 09 - Orlando 


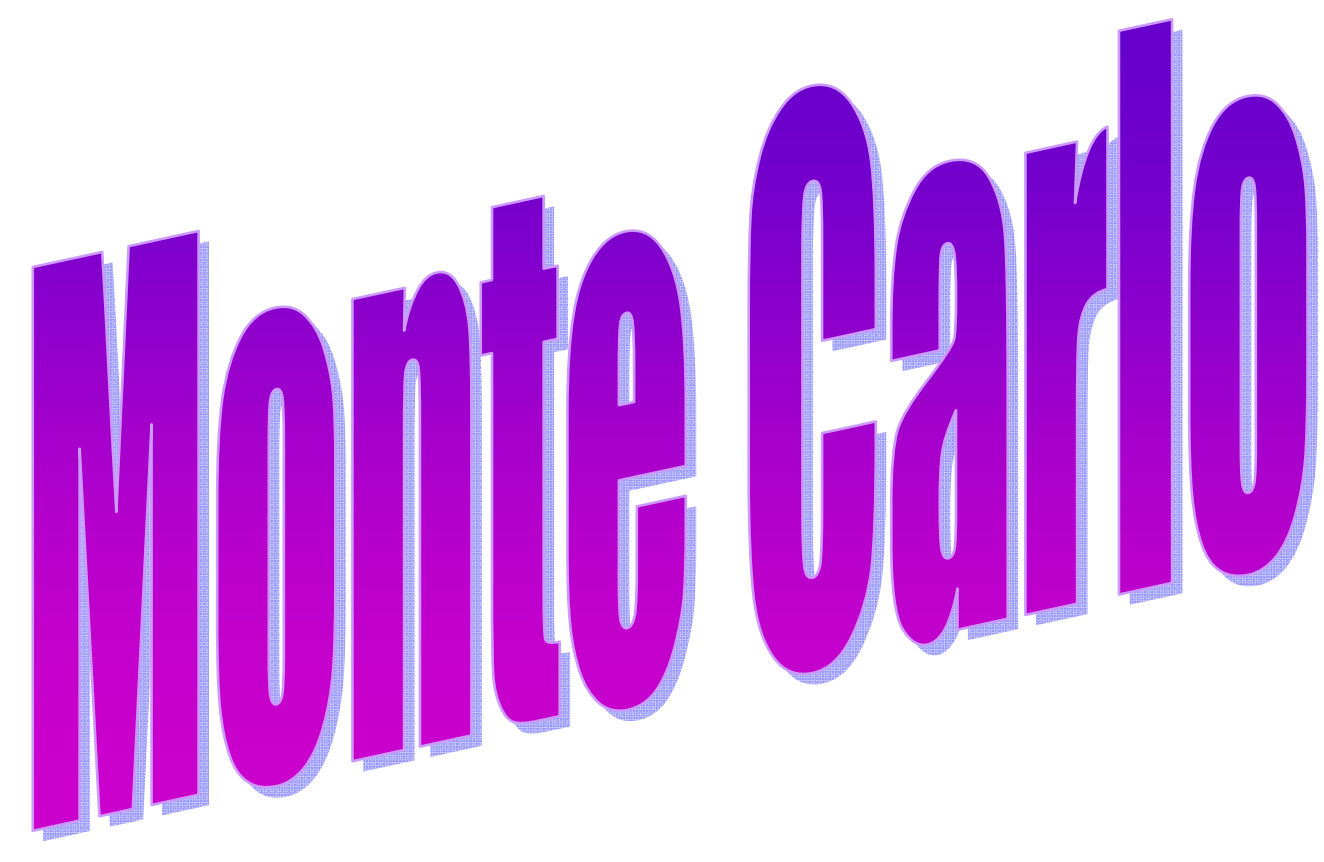

Mauro Villa - IEEE 09 - Orlando 


\section{trie mumber ot retiections II trie Cherenkov tube}

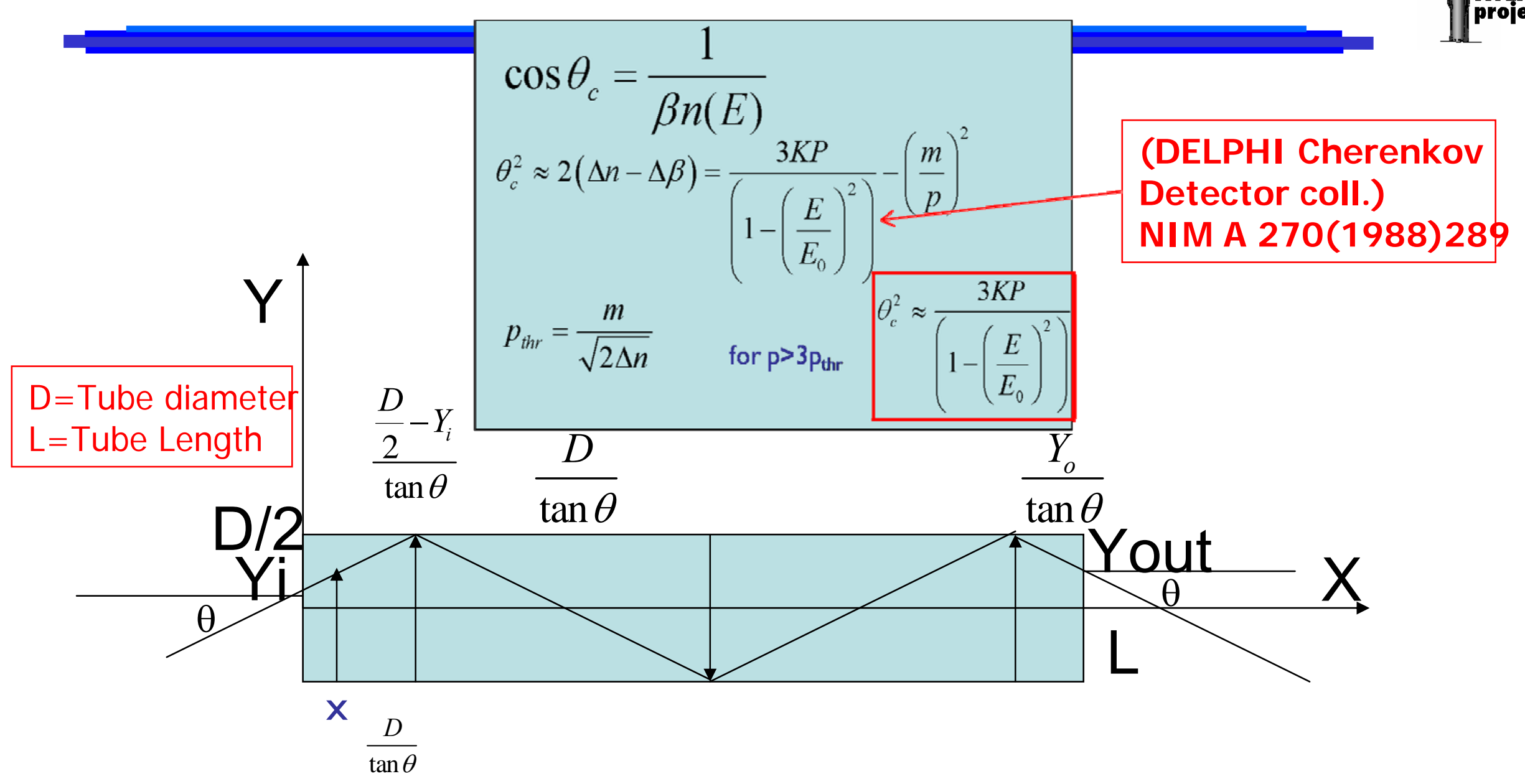

$$
\begin{array}{r}
N_{r}(E, x) \approx \frac{(L-x)}{D} \sqrt{\frac{3 K P}{1-\left(\frac{E}{E_{E}}\right)^{2}}}+\frac{Y_{i}}{D} \\
\text { Mauro Villa - }{ }_{\text {IEEE }} \text { 09 - Orlando }
\end{array}
$$

In the following we will develop all calculation for $\mathrm{Yi}=0$. 


\section{Aluminum retlectivity and Isobutane Transmittivity}

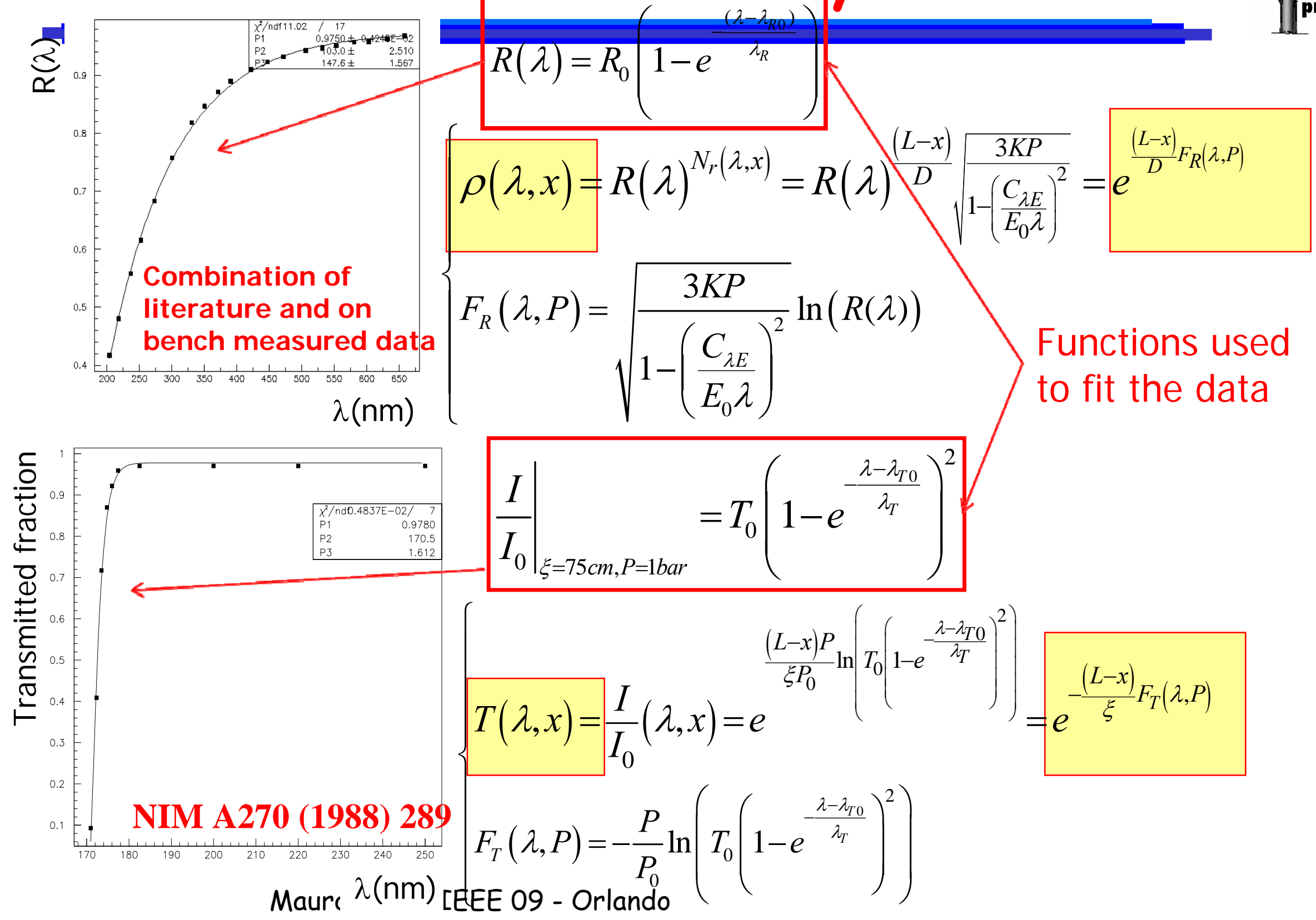




\section{uIs Irivuniturn at the tube exit}

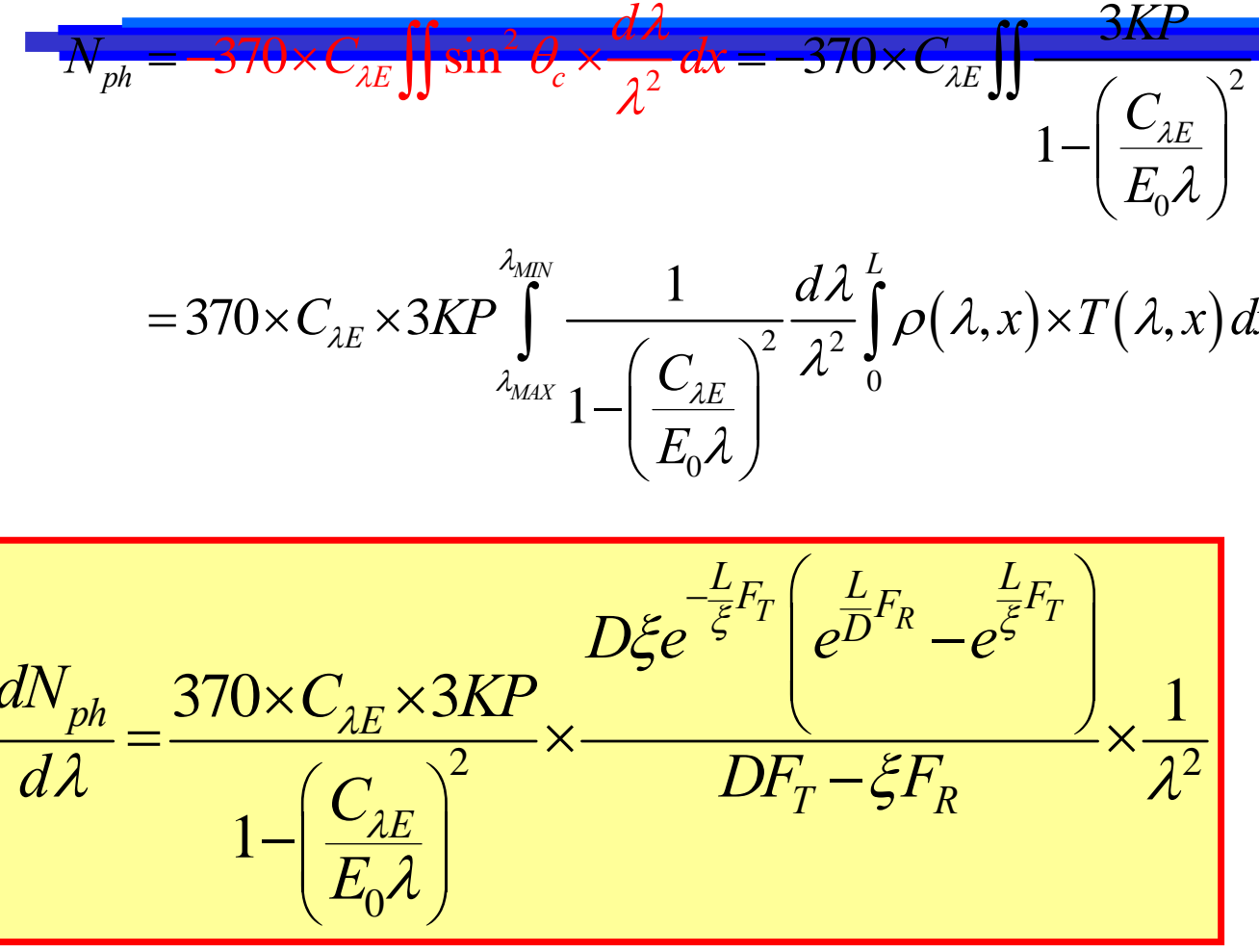

$\square$ The photomultiplier window

Quantum Efficiency can not be easily included in the model and a numerical computation is necessary

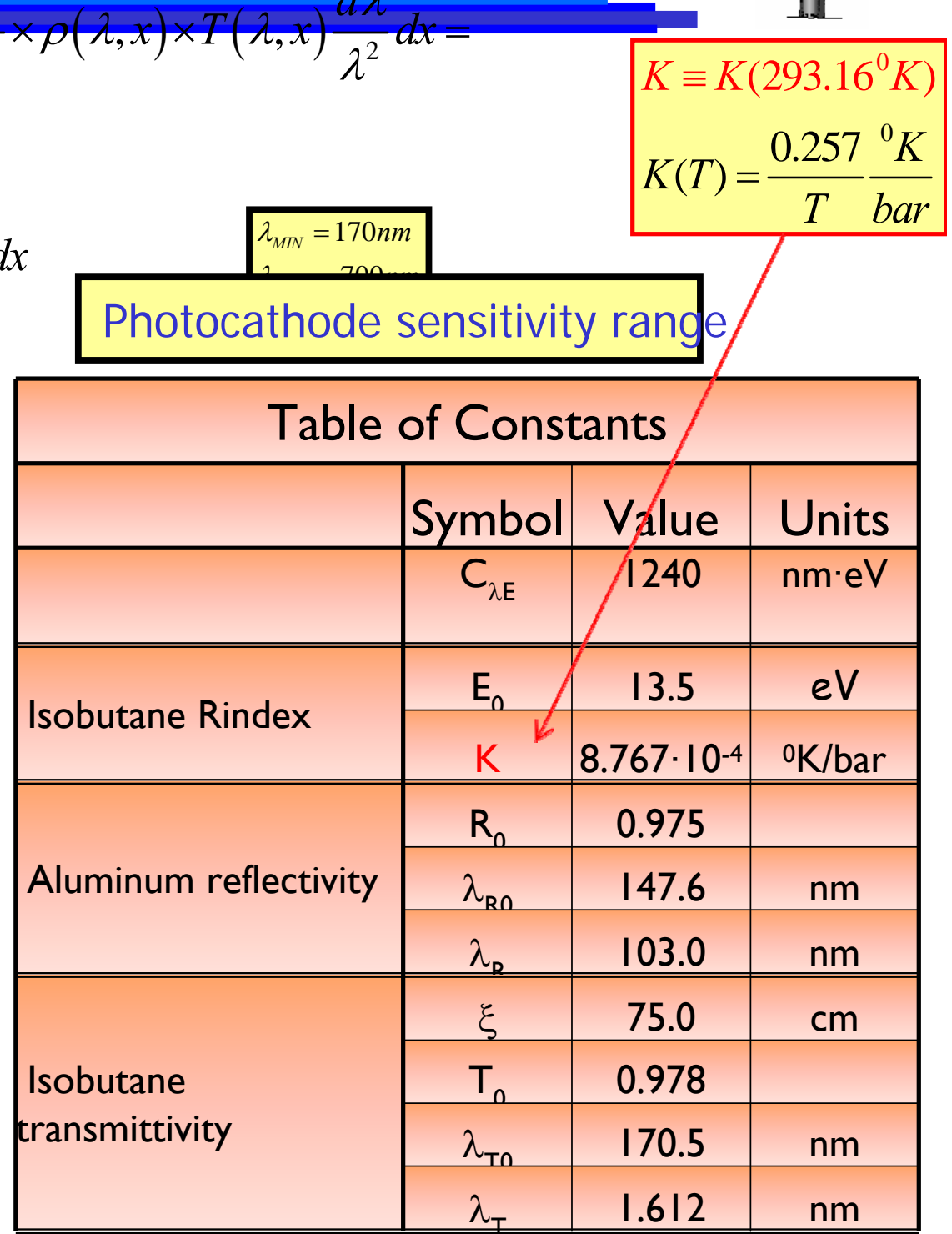




\section{simulation}

LUCID has been tested and calibrated with a beam of $180 \mathrm{GeV}$ pions (SPS H8)
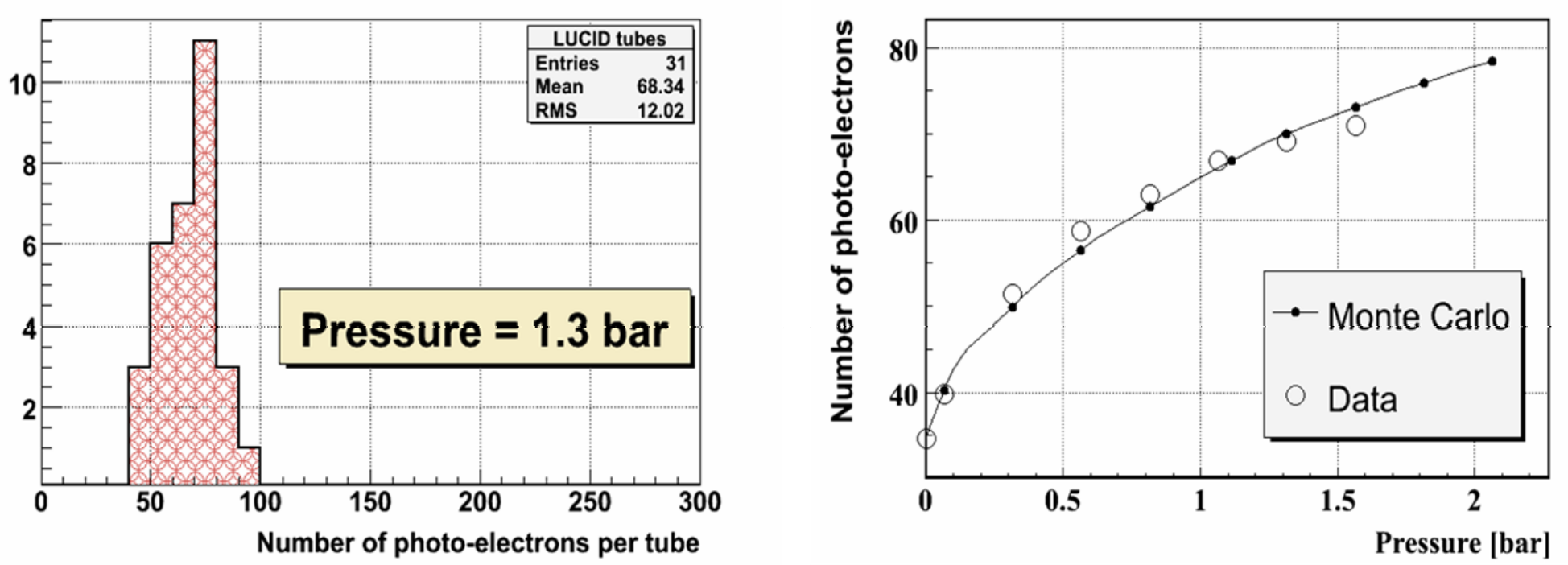

\begin{tabular}{l|l|l|l|l|l|l|l|l|l|l|} 
& P(bar) & 0.1 & 0.3 & 0.6 & 0.8 & 1.1 & 1.3 & 1.6 & 1.8 & 2.1 \\
\hline \# of & MC & 40 & 50 & 57 & 62 & 67 & 70 & 73 & 76 & 79 \\
p.e. & Model & 33 & 45 & 53 & 57 & 63 & 67 & 71 & 74 & 78 \\
\hline & $\Delta(\%)$ & 11 & 11 & 6 & 6 & 5 & 5 & 3 & 3 & 1 \\
\hline
\end{tabular}

A very good agreement is found between the photoelectron yields of this model and the LUCI D MC simulation 


\section{The gas Refractive Index}

\section{NIM A270 (1988) 289 (DELPHI Cherenkov Detector coll.)}

$$
\frac{n^{2}-1}{n^{2}+2}=\frac{0.257}{1-\left(\frac{E(e V)}{13.5}\right)^{2}} \times \frac{P(\text { bar })}{T}=\left(\text { at } T=20^{\circ} \mathrm{K}\right)=\frac{8.767 \cdot 10^{-4} P(\text { bar })}{1-\left(\frac{E(e V)}{13.5}\right)^{2}}=\frac{K P(\text { bar })}{1-\left(\frac{E(e V)}{E_{0}}\right)^{2}}
$$

$$
\begin{aligned}
& \mathrm{n}=1+\Delta \mathrm{n}, \beta=1-\Delta \beta \\
& \Delta n=\frac{\frac{3}{2} K P(\text { bar })}{1-\left(\frac{E(e V)}{E_{0}}\right)^{2}}, \quad \Delta \beta=\frac{1}{2}\left(\frac{m}{p}\right)^{2}
\end{aligned}
$$

At the production threshold the angle $\theta_{\mathbf{c}}$ is about 0

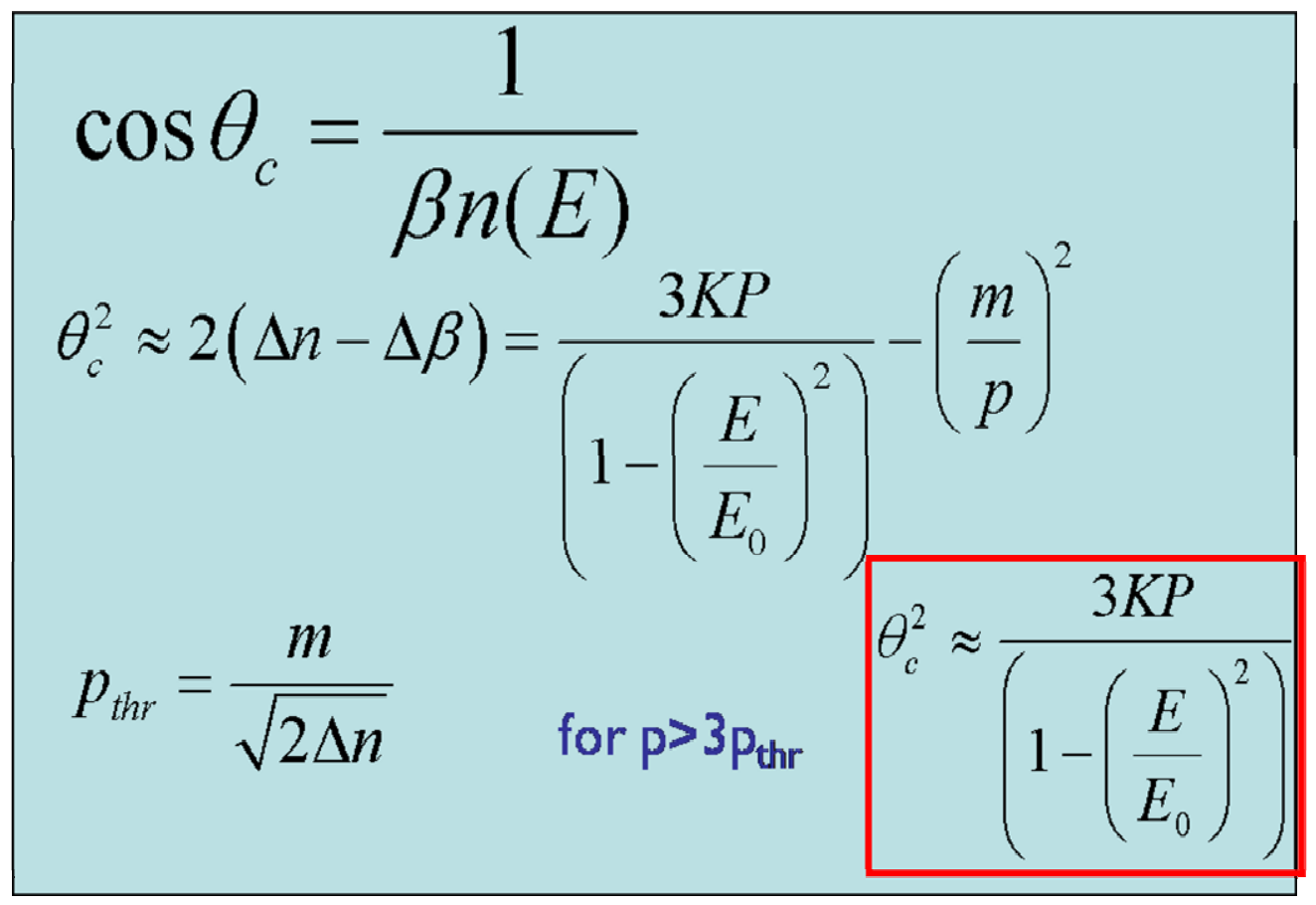

$C_{\lambda E}=\lambda(\mathrm{nm}) E(\mathrm{eV})=1240 \mathrm{~nm} \cdot \mathrm{eV}$ 


\section{The number of reflections inside a tube}

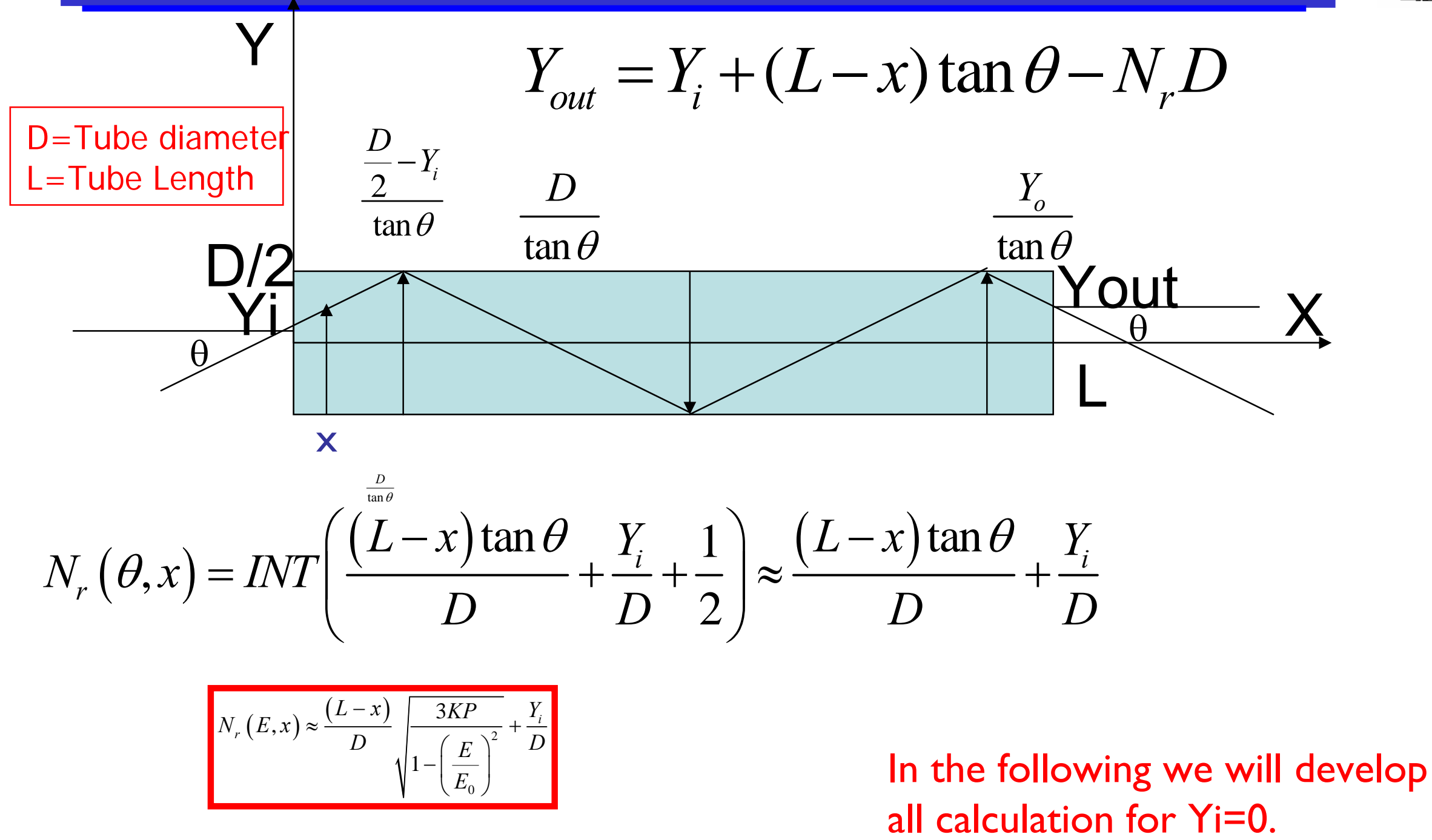




\section{Aluminum retlectivity and Isobutane Transmittivity}

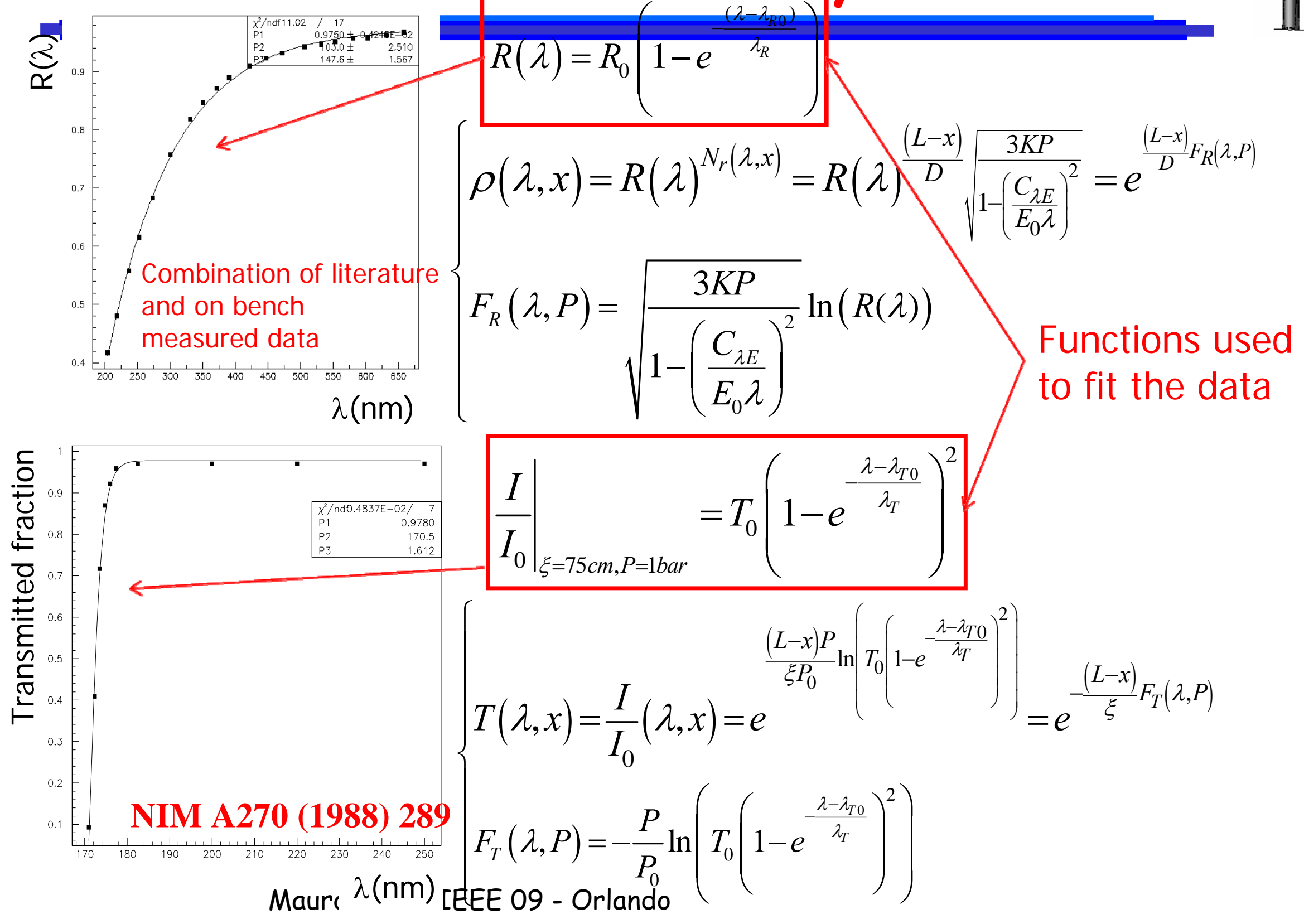


UIS ITIUU I IUTI at the tube exit

$$
\begin{aligned}
& N_{p h}=-370 \times C_{\lambda E} \iint \sin \theta_{c} \times \frac{}{\lambda^{2}} d x=-370 \times C_{\lambda E} \int \frac{3{ }_{1}}{1-\left(\frac{C_{\lambda E}}{E_{0} \lambda}\right)^{2}} \times \rho(\lambda, x) \times T(\lambda, x) \frac{d}{\lambda^{2}} d x= \\
& =370 \times C_{\lambda E} \times 3 K P \int_{\lambda_{M A X}}^{\lambda_{M I N}} \frac{1}{1-\left(\frac{C_{\lambda E}}{E_{0} \lambda}\right)^{2}} \frac{d \lambda}{\lambda^{2}} \int_{0}^{L} \rho(\lambda, x) \times T(\lambda, x) d x \\
& =370 \times C_{\lambda E} \times 3 K P \int_{\lambda_{M A X}}^{\lambda_{M N}} \frac{1}{1-\left(\frac{C_{\lambda E}}{E_{0} \lambda}\right)^{2}} \frac{d \lambda}{\lambda^{2}} \int_{0}^{L} e^{\frac{L-x}{D} F_{R}(\lambda, P)} \times e^{-\frac{L-x}{\xi} F_{T}(\lambda, P)} d x \\
& =370 \times C_{\lambda E} \times 3 K P \int_{\lambda_{M A X}}^{\lambda_{M N}} \frac{1}{1-\left(\frac{C_{\lambda E}}{E_{0} \lambda}\right)^{2}} \frac{D \xi e^{-\frac{L}{\xi} F_{T}(\lambda, P)}\left(e^{\frac{L}{D} F_{R}(\lambda, P)}-e^{\frac{L}{\xi} F_{T}(\lambda, P)}\right)}{D F_{T}(\lambda, P)-\xi F_{R}(\lambda, P)} \frac{d \lambda}{\lambda^{2}} \\
& \frac{d N_{p h}}{d \lambda}=\frac{370 \times C_{\lambda E} \times 3 K P}{1-\left(\frac{C_{\lambda E}}{E_{0} \lambda}\right)^{2}} \times \frac{D \xi e^{-\frac{L}{\xi} F_{T}}\left(e^{\frac{L}{D} F_{R}}-e^{\frac{L}{\xi} F_{T}}\right)}{D F_{T}-\xi F_{R}} \times \frac{1}{\lambda^{2}} \\
& \square \text { The photomultiplier window } \\
& \text { Quantum Efficiency can not be easily }
\end{aligned}
$$




\section{Table of constants and Quantum Efficiency typical plots}

Spectral Response Characteristics

$$
\text { Tube Type R762 }
$$

SerialNo SANPLE.A

Max. Q.E. $24.7 \%$
Wave ength of $\max . \quad 350 \mathrm{~nm}$

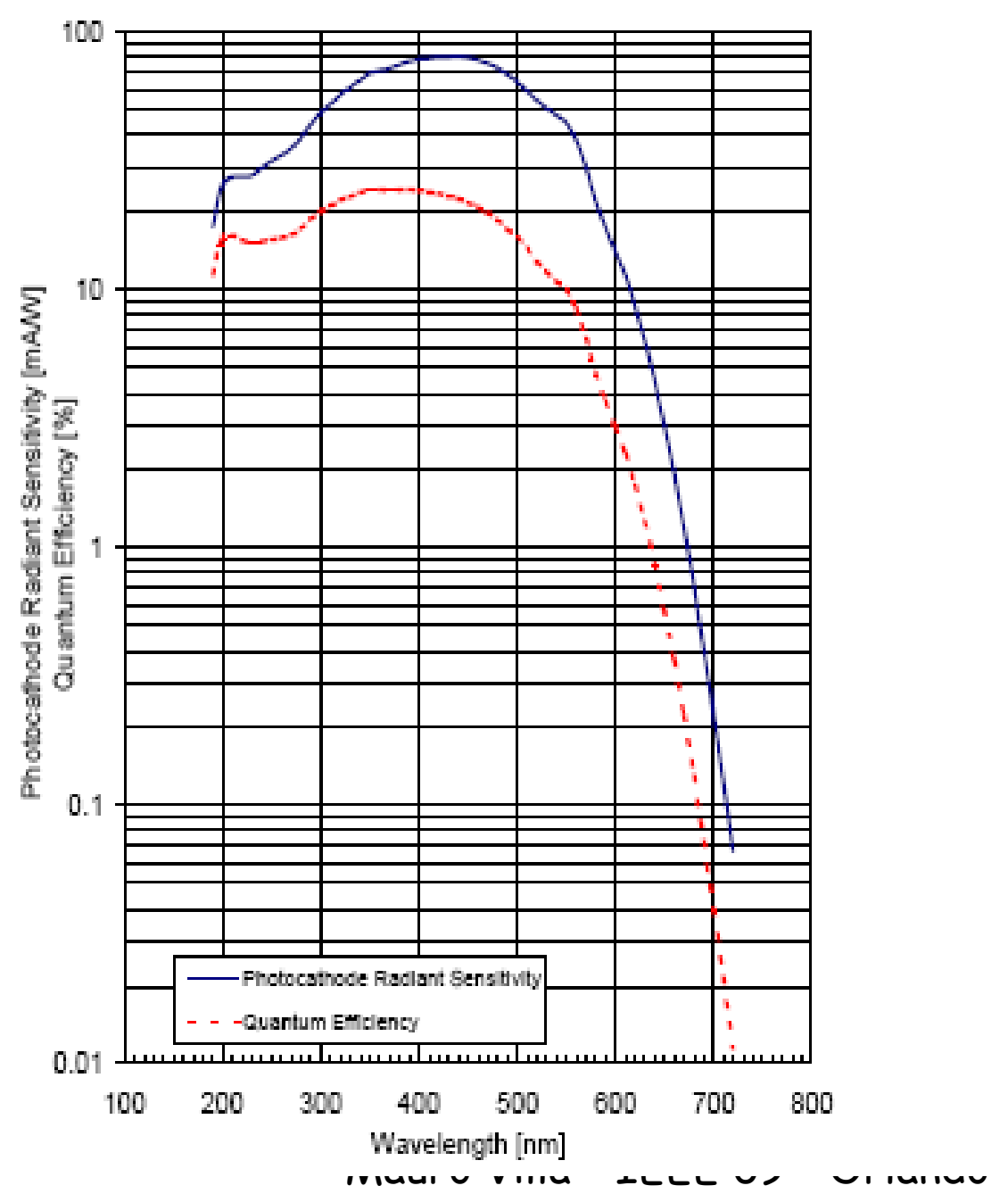

Spectral Response Characteristics

$\begin{array}{ll}\text { Tute Type } & \text { R762 } \\ \text { Serigl No. } & \text { SAMPLE-A }\end{array}$

\begin{tabular}{|c|c|c|c|}
\hline \multicolumn{4}{|c|}{ Table of Constants } \\
\hline & Symbol & Value & Units \\
\hline & $\mathrm{C}_{\lambda \mathrm{E}}$ & 1240 & $\mathrm{~nm} \cdot \mathrm{eV}$ \\
\hline \multirow{2}{*}{ Isobutane Rindex } & $\mathrm{E}_{0}$ & 13.5 & $\mathrm{eV}$ \\
\hline & K & $8.767 \cdot 10^{-4}$ & 0K/bar \\
\hline \multirow{3}{*}{ Aluminum reflectivity } & $\mathrm{R}_{0}$ & 0.975 & \\
\hline & $\lambda_{R n}$ & 147.6 & $\mathrm{~nm}$ \\
\hline & $\lambda_{R}$ & 103.0 & $\mathrm{~nm}$ \\
\hline \multirow{4}{*}{$\begin{array}{l}\text { Isobutane } \\
\text { transmittivity }\end{array}$} & $\xi$ & 75.0 & $\mathrm{~cm}$ \\
\hline & $T_{0}$ & 0.978 & \\
\hline & $\lambda_{\text {Tn }}$ & 170.5 & $\mathrm{~nm}$ \\
\hline & $\lambda_{I}$ & 1.612 & $\mathrm{~nm}$ \\
\hline & & \multicolumn{2}{|c|}{$\begin{array}{l}K \equiv K\left(293.16^{0} \mathrm{~K}\right) \\
K(T)=\frac{0.257}{T} \frac{{ }^{0} \mathrm{~K}}{b a r}\end{array}$} \\
\hline
\end{tabular}




\section{Aluminium reflectivity measurements}

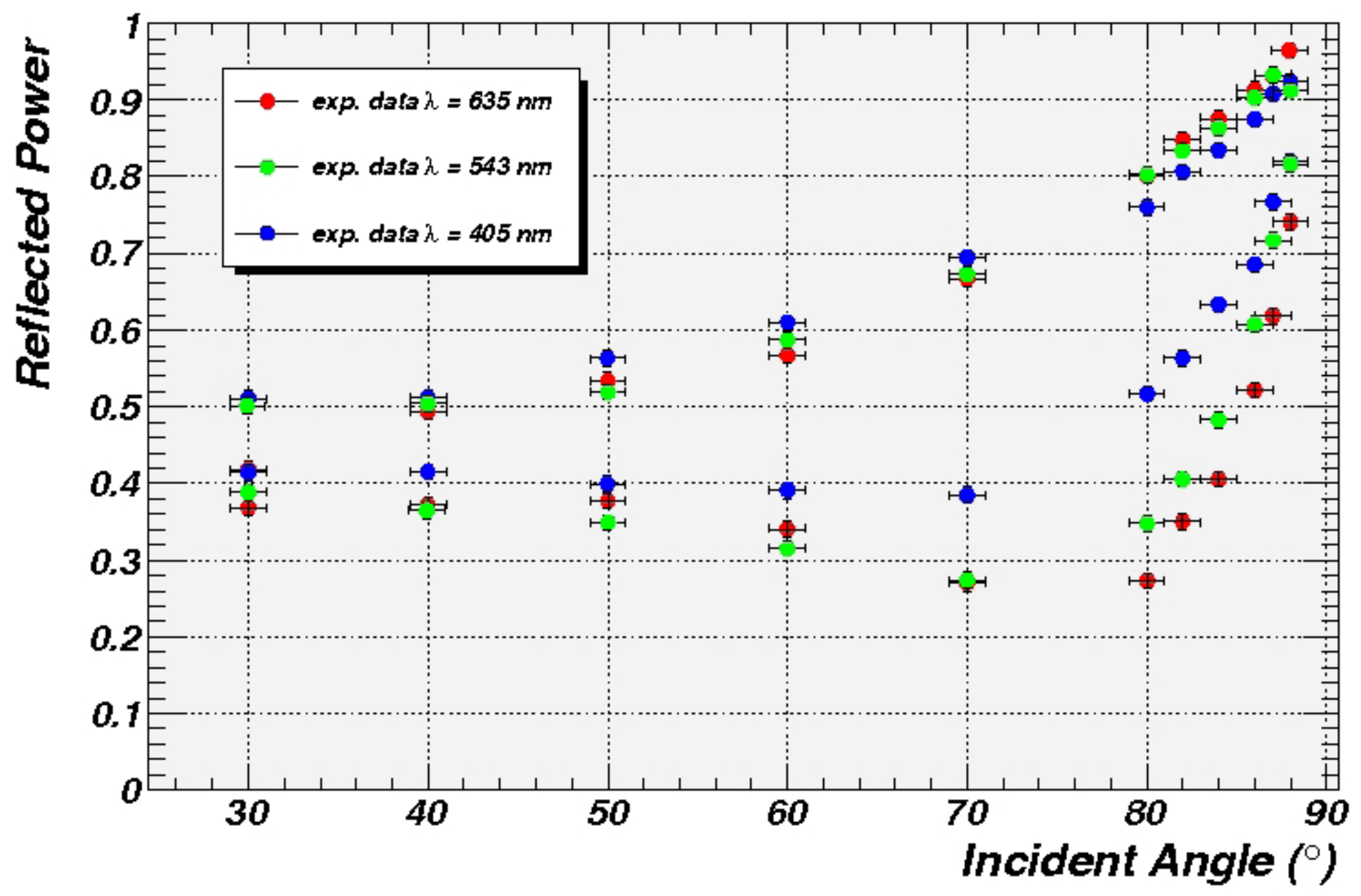

Mauro Villa - IEEE 09 - Orlando 

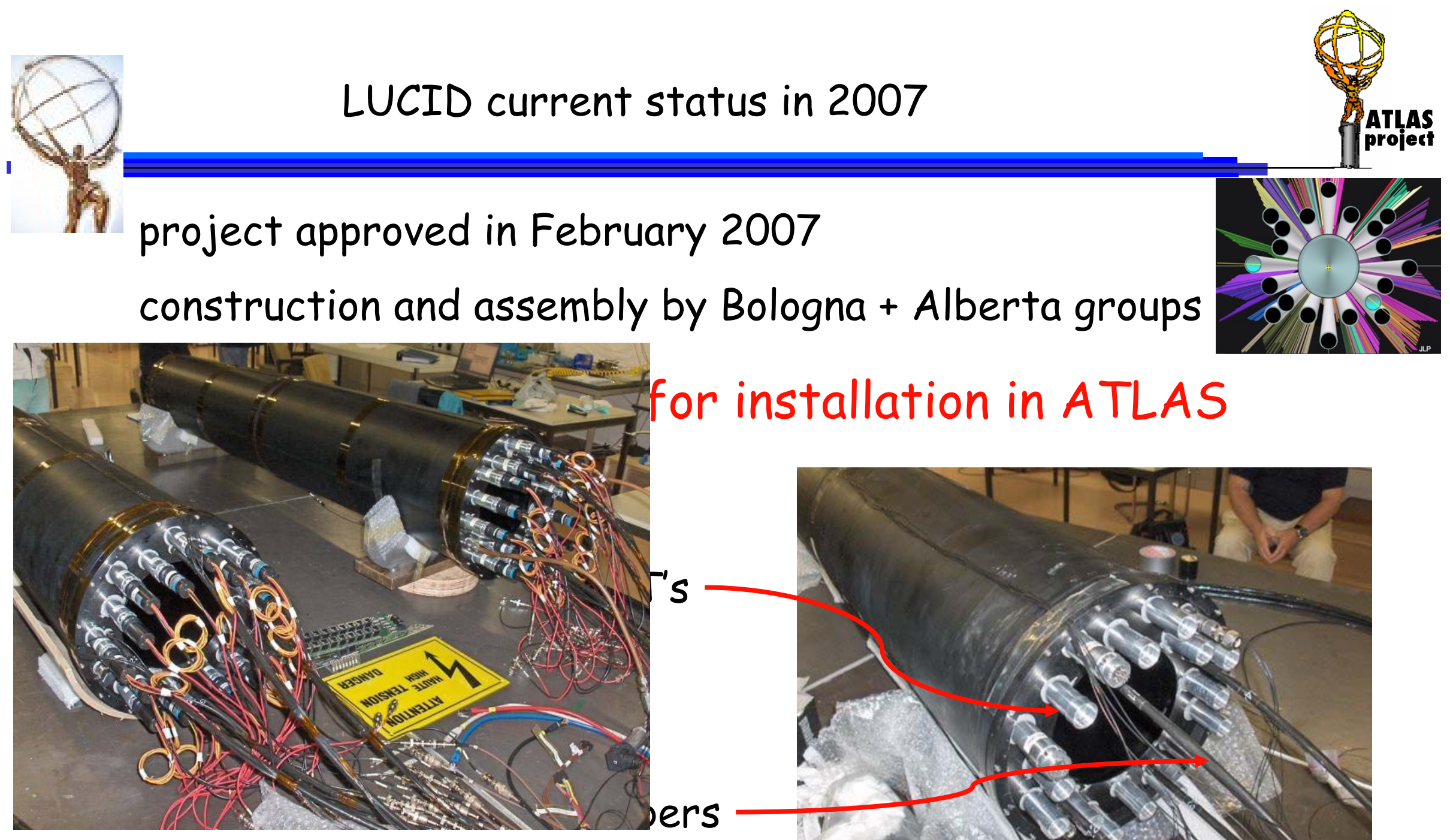

installation in ATLAS

project approved in February 2007

construction and assembly by Bologna + Alberta groups

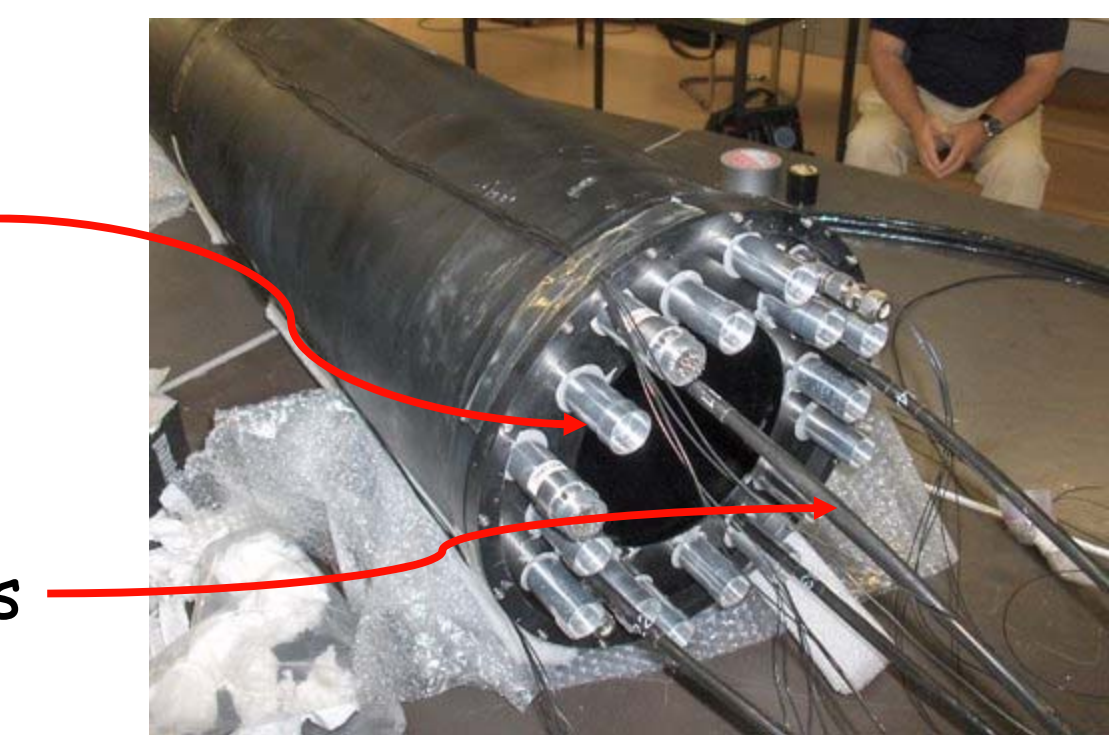

completed tests:

- vessel sealing, LED's read out, calibration 